OPEN ACCESS

Edited by:

Tim Lloyd Setter,

Cornell University, United States

Reviewed by:

Helena Storchova,

Academy of Sciences of the Czech

Republic, Czechia

Lee Jeong Hwan,

Chonbuk National University,

South Korea

*Correspondence:

Natalia Pabón-Mora

lucia.pabon@udea.edu.co

${ }^{\dagger}$ These authors have contributed equally to this work

Specialty section:

This article was submitted to Plant Development and EvoDevo, a section of the journal

Frontiers in Plant Science

Received: 23 December 2019 Accepted: 30 March 2020

Published: 21 April 2020

Citation:

Ospina-Zapata DA, Madrigal Y, Alzate JF and Pabón-Mora N (2020)

Evolution and Expression

of Reproductive Transition Regulatory

Genes FTITFL1 With Emphasis

in Selected Neotropical Orchids.

Front. Plant Sci. 11:469.

doi: 10.3389/fpls.2020.00469

\section{Evolution and Expression of Reproductive Transition Regulatory Genes FT/TFL1 With Emphasis in Selected Neotropical Orchids}

\author{
Diego A. Ospina-Zapata ${ }^{1 \dagger}$, Yesenia Madrigal'tt, Juan F. Alzate ${ }^{2}$ and \\ Natalia Pabón-Mora ${ }^{1 *}$ \\ ${ }^{1}$ Facultad de Ciencias Exactas y Naturales, Instituto de Biología, Universidad de Antioquia, Medellín, Colombia, ${ }^{2}$ Centro \\ Nacional de Secuenciación Genómica, Sede de Investigación Universitaria, Facultad de Medicina, Universidad de Antioquia, \\ Medellín, Colombia
}

Flowering is a rigorously timed and morphologically complex shift in plant development. This change depends on endogenous as well as environmental factors. FLOWERING LOCUS T (FT) integrates several cues from different pathways acting as a flowering promoter. Contrary to the role of $F T$, its paralog TERMINAL FLOWER 1 (TFL1) delays floral transition. Although FT/TFL1 homologs have been studied in model eudicots and monocots, scarce studies are available in non-model monocots like the Orchidaceae. Orchids are very diverse and their floral complexity is translated into a unique aesthetic display, which appeals the ornamental plant market. Nonetheless, orchid trade faces huge limitations due to their long vegetative phase and intractable indoor flowering seasons. Little is known about the genetic basis that control reproductive transition in orchids and, consequently, manipulating their flowering time remains a challenge. In order to contribute to the understanding of the genetic bases that control flowering in orchids we present here the first broad-scale analysis of FT/TFL1-like genes in monocots with an expanded sampling in Orchidaceae. We also compare expression patterns in three selected species and propose hypotheses on the putative role of these genes in their reproductive transition. Our findings show that FT-like genes are by far more diversified than TFL1-like genes in monocots with six subclades in the former and only one in the latter. Within MonFT1, the comparative protein sequences of MonFT1A and MonFT1B suggest that they could have recruited functional roles in delaying flowering, a role typically assigned to TFL1-like proteins. On the other hand, MonFT2 proteins have retained their canonical motifs and roles in promoting flowering transition. This is also shown by their increased expression levels from the shoot apical meristem (SAM) and leaves to inflorescence meristems (IM) and floral buds (FBs). Finally, TFL 1-like genes are retained as single copy and often times are lost. Their loss could be linked to the parallel recruitment of MonFT1A and MonFT1B homologs in delaying flowering and maintaining indeterminacy of the inflorescence meristem. These hypotheses lay the foundation for future functional validation in emerging model orchid species and comparative analyses in orchids with high horticultural potential in the market.

Keywords: flowering, FLOWERING LOCUS T, TERMINAL FLOWER 1, Orchidaceae, gene evolution 


\section{INTRODUCTION}

The transformation of a vegetative shoot apical meristem (SAM) into an inflorescence meristem (IM) forming bracts and floral buds (FBs) is a rigorously timed and morphologically complex shift in plant development (Henderson and Dean, 2004; Benlloch et al., 2007; Liu et al., 2009; Thouet et al., 2012). In the model species Arabidopsis thaliana (Brassicaceae) floral transition depends on endogenous as well as environmental factors, which have been dissected into different pathways. These include the photoperiod, vernalization, hormonal and autonomous agerelated pathways (Mouradov et al., 2002; Blázquez et al., 2003; Mutasa-Göttgens and Hedden, 2009; Amasino, 2010). All routes converge in the floral integrators, a group of genes that control the vegetative-to-reproductive transition which include FLOWERING LOCUS T (FT), SUPPRESSOR OF OVEREXPRESSION OF CONSTANS 1 (SOC1), LEAFY (LFY), FLOWERING LOCUS D (FD), and AGAMOUS like 24 (AGL24) (Boss et al., 2004; Wellmer and Riechmann, 2010).

Within the photoperiod pathway, FT plays one of the most important functions in reproductive transition. $F T$ is synthesized in the leaves and transported through the phloem, both as mRNA and protein, to the SAM, where it triggers flowering (Corbesier et al., 2007; Andrés and Coupland, 2012; Lu et al., 2012). FT overexpression results in early flowering plants with determinate inflorescences (Kardailsky et al., 1999). Conversely, ft mutant plants produce more leaves and have delayed flowering (Koornneef et al., 1991). In addition, FT is positively regulated by CONSTANS (CO), an upstream activator responsive to light exposure (Suárez-López et al., 2001; Valverde, 2011). In the SAM, FT interacts with FD and promotes the expression of APETALA 1 (AP1) and SOC1 (Abe et al., 2005; Wigge et al., 2005; Lee and Lee, 2010). In turn, SOC1 and AGL24 induce the transcription of $L F Y$ (Lee and Lee, 2010). Both $L F Y$ and AP1 control floral meristem identity directly by repressing inflorescence meristem factors (Lee and Lee, 2010). Comparative studies in model monocots like Oryza sativa have found a third protein, 14-3-3 which mediates the interaction between OsFD1 (the FD homolog) and Hd3a (the FT homolog). The complex formed by these proteins is recognized as the florigen activation complex (FAC), a direct inducer of OsMADS15 (the AP1 homolog) (Taoka et al., 2011, 2013). Contrary to the role of FT, its paralog TERMINAL FLOWER 1 (TFL1) delays floral transition (Ratcliffe et al., 1998). In Arabidopsis, overexpression of TFL1 results in delayed flowering and indeterminate inflorescences (Ratcliffe et al., 1998, 1999). On the other hand, tfll mutants exhibit short vegetative phases and develop inflorescences with terminal flowers (Shannon and Meeks-Wagner, 1991; Alvarez et al., 1992; Bradley et al., 1997; Ratcliffe et al., 1998). It has also been shown that XAANTAL2 (AGAMOUS-Like14) positively regulates TFL1 expression in the SAM to promote inflorescence identity (Pérez-Ruiz et al., 2015). Interestingly, TFL1 also interacts with $\mathrm{FD}$, resulting in the negatively regulation of $A P 1$ and LFY. As a result, during the vegetative-to-reproductive transition a negative feedback loop is maintained between the inflorescence regulator TFL1 and the floral meristem genes
AP1 and LFY (Ratcliffe et al., 1999; Conti and Bradley, 2007; Hanano and Goto, 2011).

Both FT and TFL1 are members of the PhosphatidylEthanolamine-Binding Protein (PEBP) family. In plants this gene lineage is divided into the MFT-like, FT-like, and the TFL1-like subfamilies (Hedman et al., 2009; Karlgren et al., 2011; Liu et al., 2016). In A. thaliana, several members of the PEBP family have been recognized as promoters [e.g., TWIN SISTER OF FT (TSF) and MOTHER OF FT AND TFL1 (MFT)] or repressors [ARABIDOPSIS THALIANA CENTRORADIALIS (ATC) and BROTHER OF FT AND TFL1 (BFT)] of the floral transition (Yoo et al., 2004, 2010; Yamaguchi et al., 2005; Huang N. C. et al., 2012). Comparative studies of FT/TFL1 homologs have shown that their role in regulating reproductive transition is conserved in Cucurbitales (Lin et al., 2007), Fabales (Nan et al., 2014), Gentianales (Imamura et al., 2011), Poales (Tamaki et al., 2007), Rosales (Randoux et al., 2014), and Solanales (Hayama et al., 2007). Nevertheless, other roles have also been reported for FT/TFL1 homologs including associated changes in leaf shape and size during flowering in Solanum lycopersicum (Lifschitz et al., 2014), tuber development in Solanum tuberosum (Navarro et al., 2011; Teo et al., 2017), and bulb formation in Allium cepa (Lee et al., 2013). Finally, FT-like homologs have been identified as negative regulators of flowering in Beta vulgaris, Glycine max, Helianthus annuus, Nicotiana tabacum, and Populus spp., a function more similar to the canonical role of TFL1-like than to most other FT-like genes (Blackman et al., 2010; Pin et al., 2010; Hsu et al., 2011; Harig et al., 2012; Wang et al., 2015).

Although FT/TFL1 homologs have been studied in model eudicots and monocots, few studies are available in non-model monocots like the orchids. With approximately 25,000 species, the Orchidaceae is one of the most diversified families of flowering plants. Orchid flowers exhibit predominantly bilateral symmetry as a result of the elaborated medial petal (called the lip) and the congenital fusion of one (rarely 3) stamens with the stigmas into a gynostemium (Pabón-Mora and González, 2008; Hsu et al., 2015). Such floral complexity is translated into exceedingly variable arrays of form, color and size which appeals the market for ornamental plants (Teixeira da Silva, 2013; Teixeira da Silva et al., 2014). Nonetheless, orchid sale and trade faces huge limitations due to their long vegetative phase and intractable indoor flowering seasons (Wang et al., 2017). Little is known about the role of FT and TFL1 homologs during the reproductive transition in orchids (Huang W. et al., 2012; Teixeira da Silva et al., 2014). Isolation and characterization of FT homologs have only been done in Cymbidium (Huang W. et al., 2012; Xiang et al., 2012), Dendrobium (Li et al., 2012; Wang et al., 2017), Oncidium (Hou and Yang, 2009), and Phalaenopsis (Li et al., 2014; Jang et al., 2015; Zhou et al., 2018), where they appear to positively regulate reproductive transition. For instance, heterologous expression of $F T$ orchid homologs in A. thaliana, positively regulates AP1 and promotes early flowering (Hou and Yang, 2009; Huang W. et al., 2012; Li et al., 2012; Zhou et al., 2018). The same results are found when $F T$ orchid homologs are transformed into Oryza sativa and Nicotiana tabacum (Xiang et al., 2012; Jang et al., 2015). 
The mutant phenotypes of $f t A$. thaliana plants can be partially or completely reverted by the overexpression of orchid FT homologs (Hou and Yang, 2009; Li et al., 2014; Jang et al., 2015). Endogenous experiments have only been standardized in Dendrobium, where overexpression of DOFT (the FT homolog) results in early flowering (Wang et al., 2017). Interestingly, DOFT is also involved in pseudobulb formation (Wang et al., 2017). In contrast, TFL1 homologs have been less studied when compared to FT homologs. The only functional report shows that Oncidium TFL1 can delay flowering when overexpressed in wild type $A$. thaliana plants and can fully rescue $t f l 1$ mutant phenotypes (Hou and Yang, 2009), suggesting conserved roles across TFL1 homologs.

In this context, our long-term goal is to understand the role of FT/TFL1-like homologs in the vegetative-to-flowering transition in orchids, particularly in neotropical members with diverse reproductive strategies in natural conditions. Here we present the first large scale analysis of FT/TFL1-like genes in monocots with an expanded sampling in Orchidaceae. This was done through extensive searches in public repositories as well as ten newly generated orchid transcriptomes from neotropical species. Our analyses point to more duplication events and diversification of FT homologs when compared to TFL1 genes in orchids than previously thought. Finally, we compare protein sequences and expression patterns in three selected species and present hypotheses on the putative role of these genes in their reproductive transition. Our results suggest a possible loss of TFL1 homologs and a functional shift of some FT homologs to repress flowering.

\section{MATERIALS AND METHODS}

\section{Reference Transcriptome Sequencing for Neotropical Orchidaceae}

In order to identify FT/TFL1-like genes from neotropical orchids (most with horticultural value), ten transcriptomes corresponding to: Elleanthus aurantiacus, Gomphichis scaposa, Masdevallia coccinea, M. wendlandiana, Maxillaria aurea, Miltoniopsis roezlii, Oncidium "Gower Ramsey," Oncidium "Twinkle," Stelis pusilla and Tolumnia "Cherry red x Ralph yagi" were sequenced (Supplementary Table S1). The plant material was obtained from individuals grown in nurseries or in the wild in the surroundings of Medellín, Antioquia (Colombia). The reference transcriptomes were sequenced from total RNA extracted from mixed material that included vegetative (with forming leaves) and reproductive meristems (with developing flowers), as well as leaves and pseudobulbs, when present. RNA was isolated using TRIsure (Bioline, London, United Kingdom) following the manufacturer's instructions, resuspended in ethanol $100 \%$ and shipped to the sequencing facilities. The libraries were made using the TruSeq mRNA library construction kit (Illumina, San Diego, CA, United States) and sequenced on a NovaSeq 6000 equipment (Illumina, San Diego, CA, United States) with paired end readings of 100 base pairs. The transcriptome was assembled de novo with Trinity V2 at the Centro Nacional de Secuenciación Genómica (CNSG), following the default settings and adding the Trimmomatic adapter removal step. Read cleaning was performed with prinseqlite v0.20.4 with a quality threshold of Q35 and a minimum read length of 50 bases. Contig metrics are summarized in Table $\mathbf{1 .}$

\section{Isolation of FT/TFL1-Like Homologs}

In order to evaluate the evolution of PEBP genes we generated a comprehensive sampling starting within Orchidaceae and then expanding into other monocots for an inclusive phylogenetic context. In order to isolate FT/TFL1-like homologs from Orchidaceae, searches were made using BLAST (Altschul et al., 1990) on our orchid transcriptomes as well as on public repositories available. Queries used included the MFT/FT/TFL1 reported genes for Arabidopsis thaliana (Bradley et al., 1997; Kobayashi et al., 1999) and Oncidium Gower Ramsey (Hou and Yang, 2009). Repositories used for Orchidaceae included Orchidbase 3.0 (Fu et al., 2011) ${ }^{1}$ and Orchidstra 2.0 (Chao et al., $2017)^{2}$. Additionally, some of the FT/TFL1 genes previously reported in other orchids (Hou and Yang, 2009; Huang W. et al., 2012; Xiang et al., 2012; Li et al., 2014; Jang et al., 2015; Wang et al., 2017; Zhou et al., 2018) and monocots outside of the Orchidaceae, using databases like the Rice Genome Annotation Project web $^{3}$, were included (Danilevskaya et al., 2008; Lee et al., 2013; Chaurasia et al., 2017; Leeggangers et al., 2018).

In addition to monocot genes we included the canonical Arabidopsis genes for reference and sampled representative taxa from each major angiosperm lineage namely, rosids, asterids, basal eudicots, and basal angiosperms/magnoliids. For additional sampling across angiosperms, we searched $\mathrm{NCBI}^{4}$, OneKP ${ }^{5}$, Phytometasyn ${ }^{6}$, Phytozome ${ }^{7}$, and included homologs reported by Pin et al. (2010); Hsu et al. (2011), Navarro et al. (2011), and Harig et al. (2012). We also searched for FT/TFL1-like homologs in transcriptomes available in our lab for the Magnoliids Aristolochia fimbriata and A. ringens, the monocots Cattleya trianae and Hypoxis decumbens, and the eudicots Bocconia frutescens (Papaveraceae), Brunfelsia australis, and Streptosolen jamesonii (Solanaceae) (Pabón-Mora et al., 2015; Arango-Ocampo et al., 2016; Madrigal et al., 2017; Ortiz-Ramírez et al., 2018; Suárez-Baron et al., 2019).

\section{Phylogenetic Analyses}

All the isolated sequences were compiled in Bioedit ${ }^{8}$ and cleaned manually to find the ORF and keep exclusively the CDS of all hits. The nucleotide sequences were aligned using the online version of $\mathrm{MAFFT}^{9}$ with a gap opening penalty of 3.0, an offset value of 1.0 and all other default criteria. The alignment was manually edited using the CDS and ORF. The data matrix in

\footnotetext{
${ }^{1}$ http://orchidbase.itps.ncku.edu.tw/est/home2012.aspx

${ }^{2}$ http://orchidstra2.abrc.sinica.edu.tw/orchidstra2/index.php

${ }^{3}$ http://rice.plantbiology.msu.edu/

${ }^{4}$ https://www.ncbi.nlm.nih.gov/genbank/

${ }^{5}$ https://db.cngb.org/onekp/

${ }^{6}$ https://bioinformatics.tugraz.at/phytometasyn/

${ }^{7}$ https://phytozome.jgi.doe.gov/pz/portal.html

${ }^{8}$ https://bioedit.software.informer.com/versions/

${ }^{9}$ https://mafft.cbrc.jp/alignment/server/
} 
TABLE 1 | Assembly statistics of selected orchid species with mixed transcriptomes sequenced.

\begin{tabular}{|c|c|c|c|c|c|c|}
\hline Species & $\begin{array}{l}\text { Total length of } \\
\text { sequence (bp) }\end{array}$ & $\begin{array}{c}\text { Total number of } \\
\text { sequences }\end{array}$ & $\begin{array}{c}\text { Average contig } \\
\text { length (bp) }\end{array}$ & $\begin{array}{l}\text { Largest contig } \\
\text { (bp) }\end{array}$ & N50 stats (bp) & GC $\%$ \\
\hline Elleanthus aurantiacus & 100537418 & 91814 & 1095 & 12295 & 1800 & 43.47 \\
\hline Gomphichis scaposa & 85109234 & 78810 & 1079 & 18109 & 1748 & 44.41 \\
\hline Masdevalia coccinea & 102138335 & 124799 & 818 & 15492 & 1454 & 41.11 \\
\hline Masdevalia wendlandiana & 78531621 & 75953 & 1033 & 15559 & 1682 & 41.50 \\
\hline Maxilaria aurea & 72925370 & 68647 & 1062 & 12136 & 1700 & 44.59 \\
\hline Miltoniopsis roezlii & 64965610 & 59091 & 1099 & 11852 & 1755 & 43.48 \\
\hline Oncidium "Gower Ramsey" & 69431269 & 84942 & 817 & 11821 & 1176 & 44.57 \\
\hline Oncidium "Twinkle" & 74124885 & 75431 & 982 & 11607 & 1557 & 43.04 \\
\hline Stelis pusilla & 90879205 & 109206 & 832 & 11960 & 1326 & 43.47 \\
\hline Tolumnia "Cherry red $x$ Ralph yagi" & 94692300 & 95180 & 994 & 12362 & 1586 & 42.07 \\
\hline
\end{tabular}

PHYLIP format was used for phylogenetic analyses by Maximum Likelihood (ML) using the IQ-TREE software (Nguyen et al., $2015)^{10}$. The molecular evolution model that best fit the data was calculated using the ModelFinder tool incorporated in IQ-TREE (Kalyaanamoorthy et al., 2017). The Ultrafast Bootstrap (UFBS) of 1000 pseudo-replicas also implemented in IQ-TREE was used to calculate branch support (Hoang et al., 2018). One of the MFT sequences of Amborella trichopoda (AmtrMFT1) was used as outgroup. This particular sequence has the average size for other PEBP genes (ca. $525 \mathrm{bp}$ ). The trees were visualized and edited in FigTree v1.4. $3^{11}$. In addition to the complete analysis of FT/TFL1like homologs, independent ML analyses were performed for the FT-like and TFL1-like clades, using A. trichopoda AmtrTFL1 and AmtrFT1 as outgroups, respectively.

\section{Protein Sequence Analysis}

In order to identify new protein motifs, as well as those previously reported for the FT/TFL1-like proteins, permanently translated sequences were introduced to the MEME online server with default parameters (Bailey et al., 2009) ${ }^{12}$. We included the canonical FT/TFL1 homologs from A. thaliana, selected eudicot and monocot FT/TFL1 genes reported in the literature, as well as the FT/TFL1-like homologs included in our RT-PCR expression analyses (Bradley et al., 1997; Kobayashi et al., 1999; Yoo et al., 2004, 2010; Jang et al., 2009; Pin et al., 2010; Hsu et al., 2011; Navarro et al., 2011; Harig et al., 2012; Huang N. C. et al., 2012; Lee et al., 2013; Zhou et al., 2018). Additionally, specific aminoacids have been reported as crucial for promoting or repressive functioning for FT/TFL1-like proteins in A. thaliana (Hanzawa et al., 2005; Ahn et al., 2006; Ho and Weigel, 2014). In order to test whether these positions are conserved in Orchidaceae, an alignment with MAFFT was performed on the TranslatorX web server (Abascal et al., 2010) ${ }^{13}$. Sequences analyzed include FT-like monocot sequences, the canonical sequences of $A$. thaliana (Bradley et al., 1997; Kobayashi et al., 1999; Jang et al., 2009; Yoo et al., 2010, Huang N. C. et al., 2012) and some FT-like copies with repressive function from Beta

\footnotetext{
${ }^{10} \mathrm{http}: / /$ www.iqtree.org/

${ }^{11} \mathrm{http}: / /$ tree.bio.ed.ac.uk/software/figtree/

${ }^{12} \mathrm{http}: / /$ meme-suite.org/tools/meme

${ }^{13}$ http://translatorx.co.uk/
}

vulgaris, Nicotiana tabacum, and Populus trichocarpa (Pin et al., 2010; Hsu et al., 2011; Harig et al., 2012).

\section{RT-PCR and qRT-PCR Expression Analyses}

To analyze and compare the expression patterns of the FT/TFL1like homologs identified from the newly generated Orchidaceae transcriptomes, three species were selected: Cattleya trianae, Elleanthus aurantiacus, and Gomphichis scaposa. These three species were selected because they had a low copy number of FT and TFL1 gene clades. In addition, they represent different subfamilies as well as diverse habits. Dissections of vegetative (SAM) and inflorescence (IM) meristems, flower buds (FB), leaves (L), axillary buds (AB), and pseudobulbs (PS, present only in C. trianae) were made for each species and were flash frozen in liquid nitrogen. Vegetative meristems were taken from plants exclusively producing leaves after all surrounding old and young leaves were removed. Only young leaves were collected separately. Inflorescence meristems (IM) were dissected from enlarged and thickened apices from which all visible young FBs, if present, were removed. All FBs (i.e., those that could be detected by eye) were pulled together and processed as a single sample. Axillary buds were only sampled from $C$. trianae, and they correspond to buds that are still vegetative but have the potential to form new inflorescences in the axil of the bracts.

Total RNA was extracted from each dissected tissue using TRIsure (Bioline, London, United Kingdom) as explained above and it was resuspended in $20 \mu \mathrm{l}$ of autoclaved miliQ water. The RNA was treated with DNAseI (Invitrogen, Waltham, MA, United States) and quantified with NanoDrop 2000 (Thermo Fisher Scientific, Waltham, MA, United States). A total of 3.0 $\mu \mathrm{g}$ of RNA were used as a template for cDNA synthesis using SuperScript III RT (Invitrogen, Waltham, MA, United States). For the amplification of FT/TFL1-like genes, specific primers were designed for each copy avoiding conserved domains and sometimes using the UTRs (Supplementary Table S2). Each amplification reaction incorporated $9.0 \mu \mathrm{l}$ of EconoTaq (Lucigen, Middleton, United States), $5.3 \mu \mathrm{l}$ of nuclease-free water, $1.0 \mu \mathrm{l}$ of BSA $(5 \mu \mathrm{g} / \mathrm{ml}), 1.0 \mu \mathrm{l}$ of Q solution $(5 \mu \mathrm{g} / \mathrm{ml}), 1.3 \mu \mathrm{l}$ of forward primer $(10 \mathrm{mM}), 1.3 \mu \mathrm{l}$ of reverse primer $(10 \mathrm{mM})$, $0.1 \mu \mathrm{l}$ of $\mathrm{MgCl} 2$ and $1.0 \mu \mathrm{l}$ of template cDNA, for a total of 
$20 \mu \mathrm{l}$. The genes were amplified by Touchdown PCR (TD-PCR) (Korbie and Mattick, 2008), following the next thermal profiles: an initial denaturation step $\left(94^{\circ} \mathrm{C}\right.$ for $\left.40 \mathrm{~s}\right)$, an annealing step $\left(45-54^{\circ} \mathrm{C}\right.$ for $\left.40 \mathrm{~s}\right)$ and one extension step $\left(72^{\circ} \mathrm{C}\right.$ for $\left.40 \mathrm{~s}\right)$. Each TD-PCR was carried out in two phases, the initial one began with an annealing temperature $(\mathrm{Tm}) 8^{\circ} \mathrm{C}$ above the $\mathrm{Tm}$ calculated for the primers used, which then decreased $1^{\circ} \mathrm{C}$ per cycle until reaching $2^{\circ} \mathrm{C}$ below of the original $\mathrm{Tm}$, for a total of 10 cycles; and a second amplification phase of 20 cycles, using $2^{\circ} \mathrm{C}$ below the original $\mathrm{Tm}$. In total, 30 cycles per reaction were performed. Because amplification was still low and very restricted, a second experiment was carried out using 42 cycles, changing the second phase from 20 to 32 cycles. ACTIN was used as a positive control. The amplicons were visualized on $1.5 \%$ agarose gel with ethidium bromide and digitally photographed using a Whatman Biometra ${ }^{\circledR}$ BioDoc Analyzer.

To validate the expression patterns detected by RT-PCR for FT/TFL1-like homolgs, qRT-PCR assays were performed in Cattleya trianae dissected tissues. The same dissections, as well as protocols for RNA extraction and cDNA synthesis described above were used. However, $2 \mu \mathrm{g}$ of RNA were used as a template for cDNA synthesis. The $\mathrm{qPCR}$ master mix was prepared using Maxima SYBRGreen/ROX qPCR Master Mix K0222 (Waltham, MA, United States). cDNA was diluted 1:2. Three technical replicates were performed. Specific primers were designed for qRT-PCR (Supplementary Table S2). The thermal cycling regime consisted of one initial step at $95^{\circ} \mathrm{C}$ for $3 \mathrm{~min}$, then 55 cycles at $95^{\circ} \mathrm{C}$ for $5 \mathrm{~s}, 54^{\circ} \mathrm{C}$ for $5 \mathrm{~s}$, and finally $72^{\circ} \mathrm{C}$ for $20 \mathrm{~s}$ in a qTOWER3 G Real-Time Thermocycler (Analytik Jena, Jena, Germany). Endogenous genes tested included ACTIN, GADPH and 18S. Transcript levels for FT/TFL1-like genes were calculated implementing the $2^{-\Delta \Delta \mathrm{Ct}}$ function using $18 s$ as the endogenous control (Livak and Schmittgen, 2001).

\section{Scanning Electron Microscopy}

SAM, IM, and FBs from E. aurantiacus and G. scaposa were collected in $70 \%$ ethanol and stored for 1 month or longer. Apices and buds were dissected in ethanol $90 \%$. The dissected samples were dehydrated in a progressive ethanol series. Samples were critical point-dried using a Baltec CPD 030 and coated with pure gold using an Emitech K550 sputter coater. Finally, all samples were examined and photographed at $10 \mathrm{kV}$ on a Zeiss SUPRA 40VP scanning electron microscope.

\section{RESULTS}

\section{Evolution of PEBP Genes}

A comprehensive search of PEBP homologs in databases available resulted in 525 PEBP genes belonging to 101 angiosperms (Supplementary Table S3). Such comprehensive sampling resulted in 24 sequences of 9 Amborellaceae/Nymphales/Austobaileyales (ANA)/Magnoliid species, 147 genes of 25 eudicot species and 354 genes from 67 monocot species. Specifically, monocot sampling included 130 sequences of 15 non-Orchidaceae species and 224 of 52 species belonging to Orchidaceae.
Complete nucleotide sequences of all isolated homologs were used in the Maximum Likelihood (ML) analysis. The aligned matrix had a total of 1784 characters. The MFT homolog of Amborella trichopoda (AmtrMFT1) was used as outgroup. The resulting topology shows a duplication event prior to angiosperm diversification (UFBS $=100$ ), which results in the FT-like $(\mathrm{UFBS}=100)$ and BFT/TFL1-like $(\mathrm{UFBS}=100)$ clades (Supplementary Figure S1). The MFT-like copies are not clustered together but rather form the MFT-like grade, which predates the FT/TFL1-like duplication (Supplementary Figure S1). Within this grade MFT1 and MFT2-like groups were labeled (Drabešová et al., 2016). Because of the large number of sequences and the divergence between the FT and the BFT/TFL1like copies, subsequent independent ML analyses for each clade were performed. For these analyses the Amborella trichopoda AmtrTFL1 and AmtrFT1 were used as outgroups in the separate FT-like and BFT/TFL1-like analyses, respectively.

\section{FT-Like Gene Evolution}

Our sampling includes 81 sequences from 15 non-Orchidaceae monocot species and 192 sequences from 48 Orchidaceae species belonging to different subfamilies, as follows: 146 sequences from 32 species of Epidendroideae, 16 from seven species of Orchidoideae, four from three species of Cypripedioideae, 16 belonging to two species of Vanilloideae and ten of four species of Apostasioideae. Finally, 62 homologs of 19 eudicot species and five of three ANA/Magnoliid species were included. The matrix compiled included a total of 340 sequences and an alignment with 1269 characters. The topology of the ML analysis shows a duplication prior to the diversification of monocots and eudicots, resulting in the FT1 and FT2 clades (UFBS = 97) (Figures 1-3). Although there are three FT paralogs in A. trichopoda, they do not cluster with FT1 or FT2, and appear to be species-specific.

The FT1 homologs from magnoliids and eudicots form a clade $($ UFBS $=91)$ (Figure 1). With our sampling, additional duplications were traced at a family level in Solanaceae. Similarly, local species-specific duplications were found in Aquilegia coerulea, Arabidopsis thaliana, Beta vulgaris, Brunfelsia australis, Fragaria vesca, Gentiana triflora, Medicago truncatula, Nicotiana tabacum, Papaver setigerum, and Populus trichocarpa. No FT2 homologs were recovered from any magnoliid or eudicot species.

Monocot FT genes separate into two clades, MonFT1 and MonFT2, coinciding with the duplication described above. The MonFT1 genes have undergone at least two additional duplications, resulting in the MonFT1A (UFBS = 96), MonFT1B $($ UFBS $=100)$, and MonFT1C $($ UFBS $=99)$ clades (Figure 2). Within the MonFT1A clade, an additional duplication prior to diversification of all monocots was detected, but only one of the resulting copies was retained in orchids. The MonFT1B genes have undergone independent duplications in Orchidaceae and Poaceae. Specifically, our analysis identified at least one duplication in orchids and three additional duplications before the diversification of Poaceae in this subclade. Conversely, the MonFT1C copy is duplicated only in Phalaenopsis (Epidendroideae, Orchidaceae). Species-specific duplications of MonFT1 have occurred in Allium cepa, Dendrobium catenatum, Elleanthus aurantiacus, Masdevallia wendlandiana, Maxillaria 


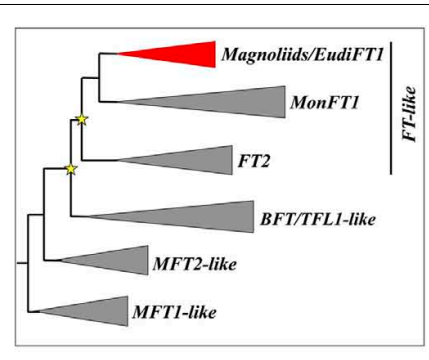

Core Eudicots

- Basal Eudicots

- Basal Angiosperms/Magnoliids

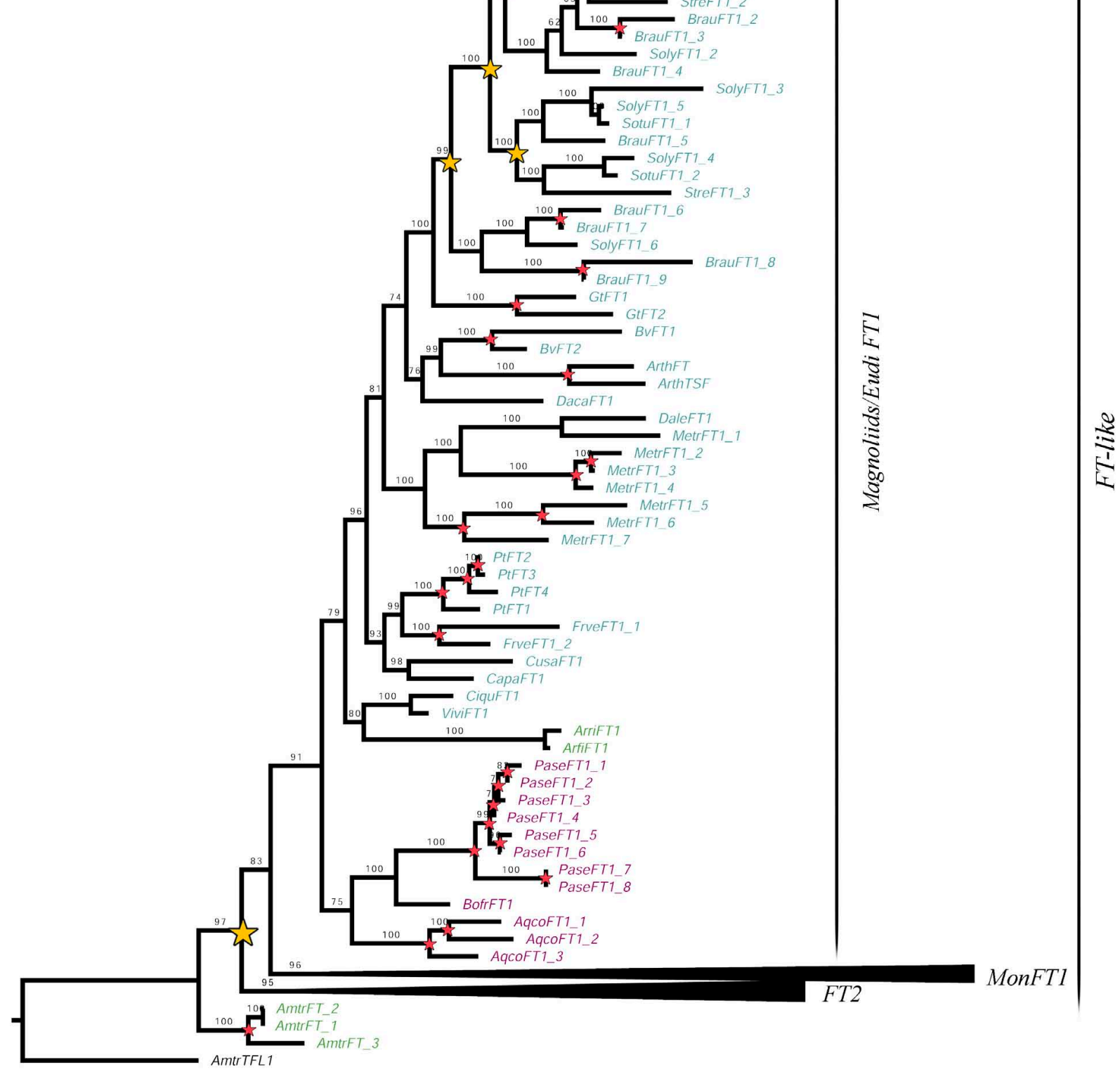

0.2

FIGURE 1 | ML analysis of Magnoliids/EudiFT1 genes. Summary tree (upper left), the expanded clade in the figure is indicated in red. Yellow stars indicate large-scale duplication events, while red stars represent intra-specific duplications. Numbers on each node indicate the Ultrafast Bootstrap (UFB) values. The collapsed clades correspond to MonFT1 (Figure 2) and FT2 (Figure 3). The colors correspond to the conventions on the left. Scale: 0.2. 

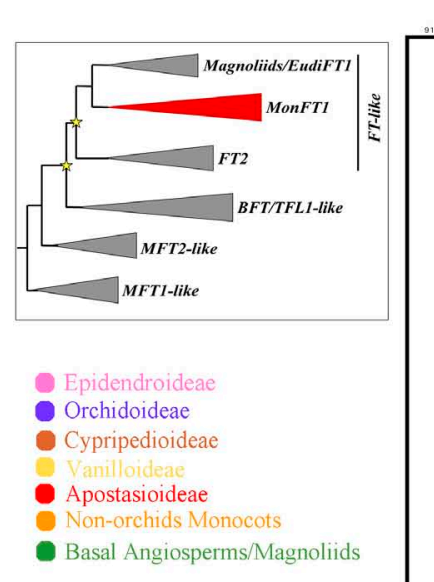

Magnoliids EudiFTI

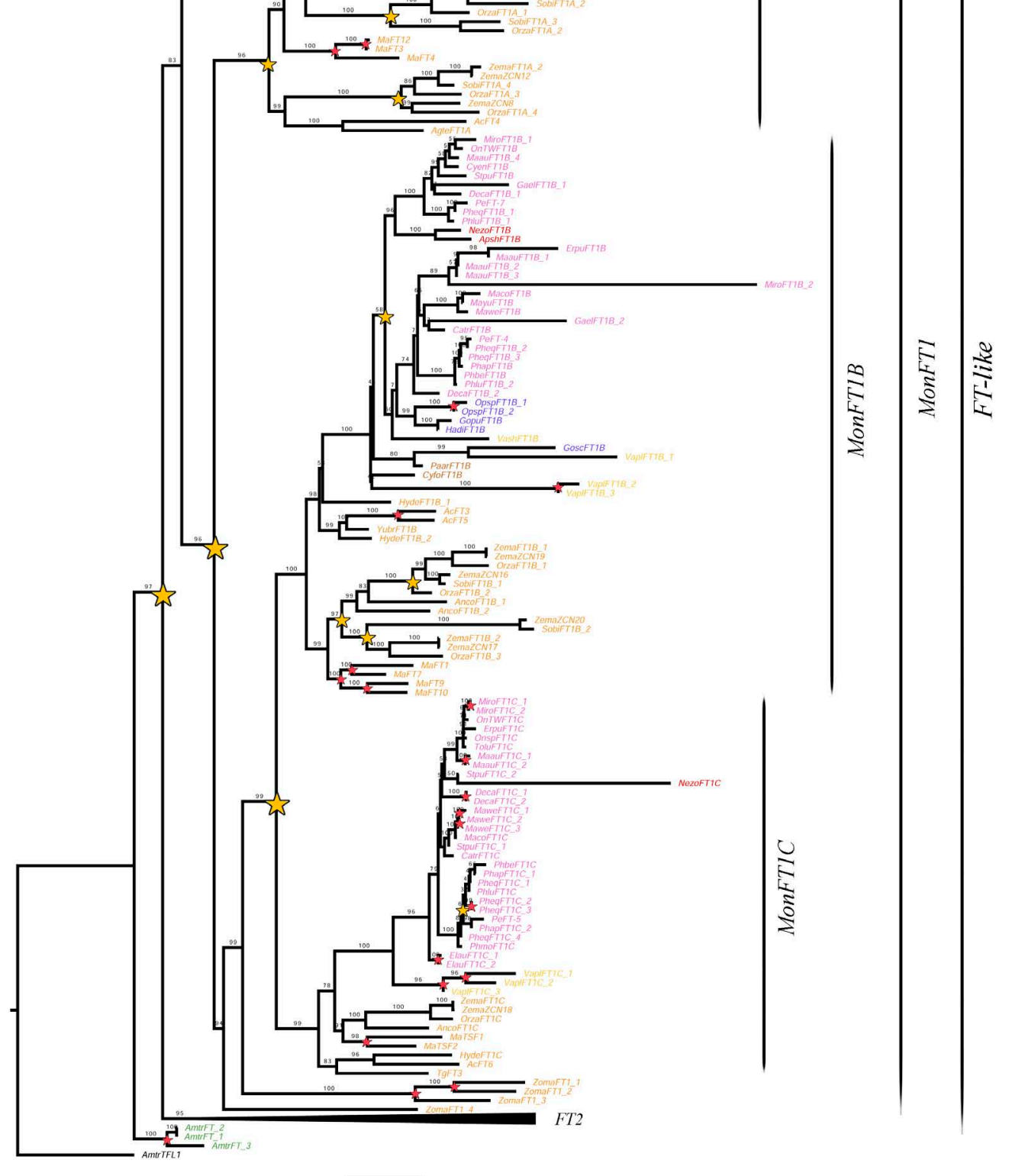

FIGURE 2 | ML analysis of MonFT1 genes. Summary tree (upper left), the expanded clade in the figure is indicated in red. Star, branch values and color conventions follow those in Figure 1. The collapsed clades correspond to Magnoliids/EudiFT1 (Figure 1) and FT2 (Figure 3). Scale: 0.2. 

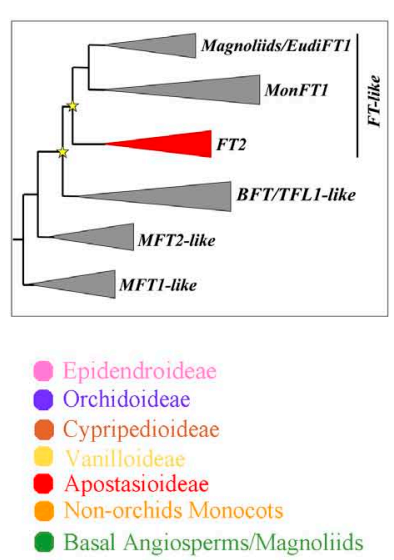

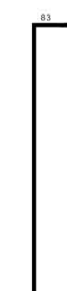
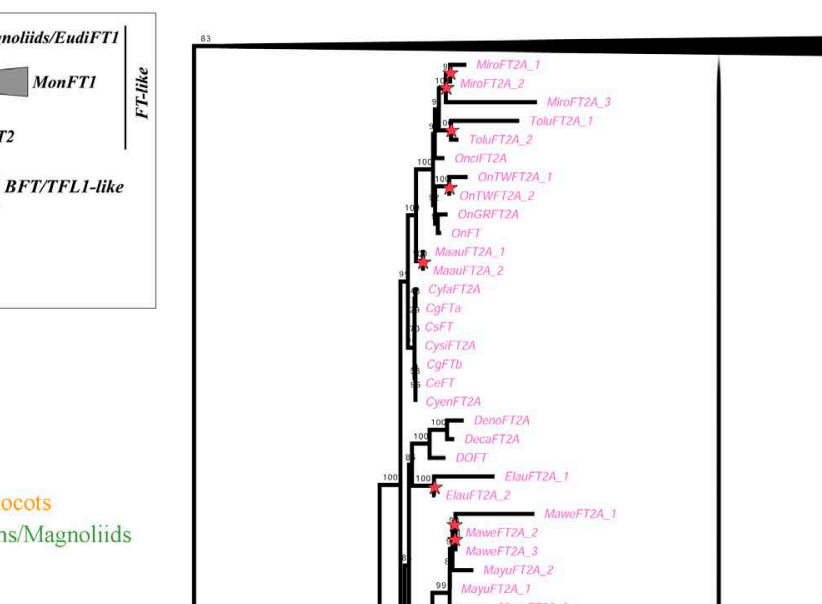

\section{FTI}

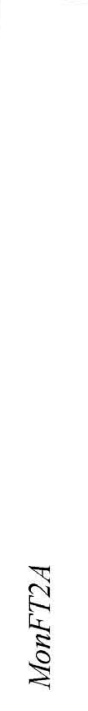

过

$\frac{8}{i=1}$
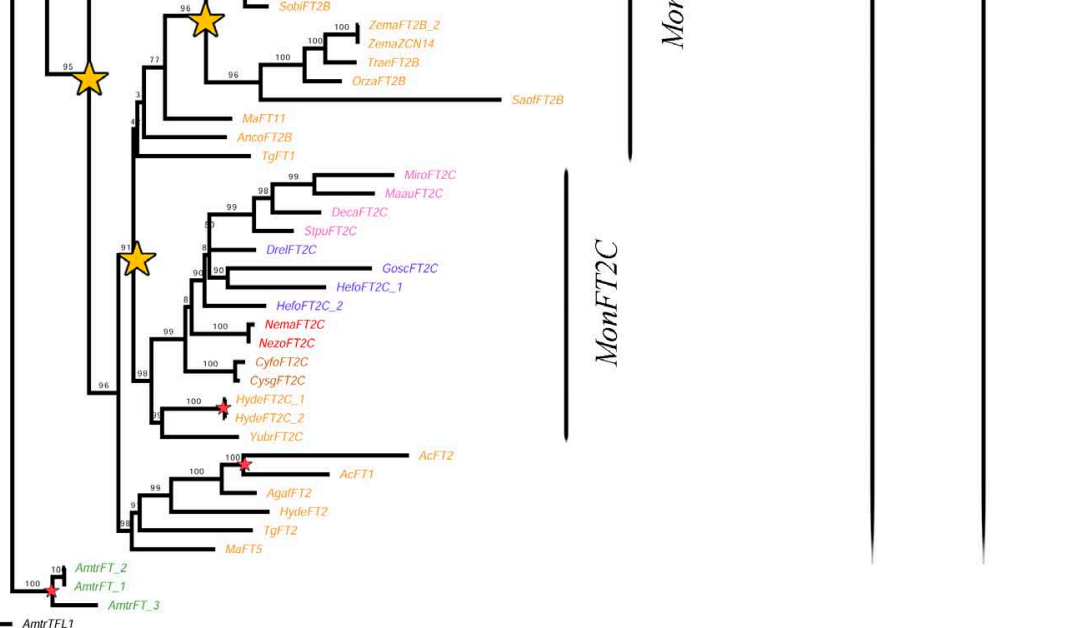

FIGURE 3 | ML analysis of MonFT2 genes. Summary tree (upper left), the expanded clade in the figure is indicated in red. Stars, branch values and color conventions follow those in Figure 1. The collapsed clade corresponds to FT1 (Figures 1, 2). Scale: 0.2. 
aurea, Miltoniopsis roezlii, Musa acuminata, Ophrys sphegodes, Phalaenopsis lueddemanniana, P. equestris, Vanilla planifolia, and Zostera marina.

As previously explained, the FT2 genes appear to be exclusive to monocots (Figure 3). Within monocots, these genes were duplicated at least twice (UFBS $=95$ and 91 respectively) resulting in the MonFT2A (UFBS = 68), MonFT2B (UFBS = 42), and MonFT2C (UFBS = 98) paralogs. The position of the Allium cepa AcFT1 and AcFT2, the Agapanthus africanus AgafFT2, the Ananas comosus AncoFT2B, the Hypoxis decumbens HydeFT2, the Musa acuminata MaFT5 and MaFT11, and the Tulipa gesneriana TgFT1 and TgFT2 homologs supports the timing of the duplication prior to monocot diversification. Additional large-scale duplications were only found in the MonFT2B clade, which was duplicated once prior to the diversification of Poaceae and Orchidaceae (UFBS =96) with the retention of one of the copies in Orchidaceae, specifically in the Epidendroideae subfamily. Finally, species-specific duplications were found in Allium cepa, Ananas comosus, Apostasia wallichii, Elleanthus aurantiacus, Gomphichis scaposa, Hypoxis decumbens, Masdevallia coccinea, Masdevallia wendlandiana, Maxillaria aurea, Miltoniopsis roezlii, Oncidium "Twinkle," Oryza sativa, Stelis pusilla, Tolumnia "Cherry red x Ralph yagi" and Vanilla planifolia.

\section{BFT/TFL1-Like Gene Evolution}

Despite the exhaustive sampling only 110 BFT/TFL1-like homologs were isolated, which account for one fifth of the total sampling. Our sampling includes five genes of Epidendroideae (5 spp.), three genes of Orchidoideae (3 spp.), one gene of Cypripedioideae (1 sp.), one gene of Apostasioideae (1 sp.), four genes of Vanilloideae ( $3 \mathrm{spp}$.), as well as 31 genes of 11 nonOrchidaceae monocots, 60 of 18 eudicots and five from three early divergent angiosperms. The aligned matrix includes 854 characters. ML topology shows a duplication resulting in the BFT-like and TFL1-like clades (UFBS =95) (Figure 4). Because the single copy of the Amborella trichopoda AmtrTFL1 does not nest within either clade, it is likely that the duplication predates the diversification of magnoliids, monocots and eudicots. In addition, a second duplication (UFBS $=96$ ) occurs in eudicots, resulting in the EudiTFL1-like and EudiCEN-like clades. The EudiCEN-like genes are present in all eudicots whereas the EudiTFL1-like genes are only found in the super-rosids. This could be explained by two alternative hypotheses: (1) the EudiTFL1-like copy was lost in basal eudicots and in Asterids; or (2) the topology may change with expanded sampling, thus the sequences of early divergent eudicots inside EudiCEN-like belong in fact to EudiTFL1-like, as indicated by the relatively low supports in these branches (UFBS $=78$ ).

Monocot sequences clustered together in a clade here called MonTFL1-like (Figure 4), in which an additional duplication can be traced after the divergence of the Alismatales (UFBS = 77). Only one of the copies is subsequently retained in the Orchidaceae. Within Poaceae two additional duplication events can be identified, one prior to the diversification of the family while other specific to the Panicoideae (Figure 4). Finally, although the BFT clade has been retained largely as single copy, at least one duplication has occurred in Solanaceae. Speciesspecific duplications in the BFT/TFL1 clade can be observed in Aquilegia coerulea, Daucus carota, Medicago truncatula, Musa acuminata, Nicotiana tabacum, Oryza sativa, Papaver setigerum, Populus trichocarpa, Solanum lycopersicum, Vitis vinifera, and Zostera marina.

\section{Sequence Analysis}

In order to identify new protein motifs, as well as those previously reported for PEBP proteins, an analysis was performed with selected sequences on the MEME server. This analysis included the permanently translated sequences from orchid species used in expression analyses (see below) and some of the previously reported sequences with expression or functional data available. We found that motifs $1-6$ are present in most of the homologs analyzed, while all other motifs are only found in some proteins (Supplementary Figure S2). For example, motif 7 is exclusive of Phalaenopsis equestris PeFT2 and PeFT-5, motifs 8, 12, and 13 are only present in some sequences belonging to the MonFT2 clade, motifs 9 and 14 are characteristic of some proteins of BFT/TFL1 clade and motif 10 is shared between Elleanthus aurantiacus ElauFT2A1 and PeFT-5. Finally, motif 11 is only present in ArthATC, ArthTFL1, and PeFT-5.

Other aminoacids have been assigned with specific roles for FT and TFL1 proteins. For instance, in A. thaliana, the FT Tyr-85 (Y) and the TFL1 His-88 $(\mathrm{H})$ homologous amino acids are important to confer part of the characteristic function of each protein, that is to promote or repress flowering, respectively (Hanzawa et al., 2005). The Tyr-85 and His- 88 are located in motif 3 (Supplementary Figure S2). Same positions were evaluated across FT-like protein monocot homologs, and it was found that some of the MonFT1B sequences possess the characteristic $\mathrm{H}$ from TFL1 instead of the expected Y from FT (Figures 5A,B).

Functional analyses using protein chimeras have also identified that peptide sequence of segments $B$ and $C$ in the FT and TFL1 proteins of $A$. thaliana are responsible for the role of each protein to promote or delay flowering (Ahn et al., 2006). Segments B and C include regions from motifs 4, 5, and 6 (Supplementary Figure S2). The amino acid sequence of segment B is conserved among FT-like proteins, whereas in TFL1-like proteins this segment evolves rapidly and therefore is variable between close homologs (Ahn et al., 2006). When segment B is compared to FT-like homologs isolated from monocots, we find that it is highly conserved in all MonFT clades except for the proteins belonging to the MonFT1A clade (Figures 5C,D and Supplementary Figure S3). These sequences are highly variable and, thus, they resemble TFL1-like proteins (Figures 5C,D; Ahn et al., 2006). Inside segment B, a residue has been identified in the homologous position Gln-140 (Q) and Asp-144 (D) to distinguish FT from TFL1 in A. thaliana respectively (Ahn et al., 2006). Our sequence analysis revealed that in members of the MonFT1A clade the Gln (Q) is replaced by Glu (E) or His (H) (Figure 5D).

On the other hand, segment $\mathrm{C}$ is very similar between FT and TFL1 proteins. However, a few amino acids located between the homolog positions $150-152$ and 154-156 can be 

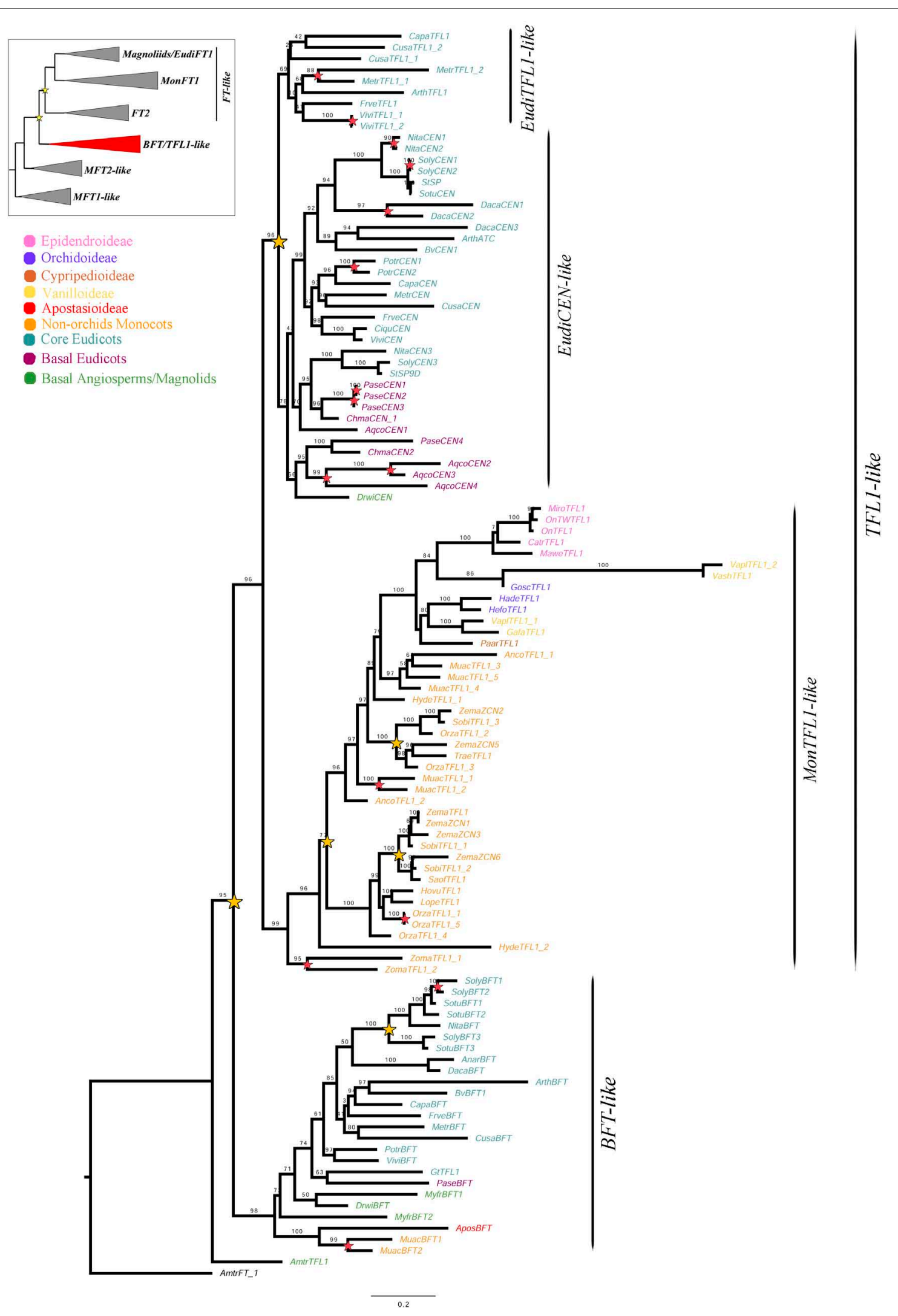

FIGURE 4 | ML analysis of BFT/TFL1-like genes. Summary tree (upper left), the expanded clade in the figure is indicated in red. Star, branch values and color conventions follow those in Figure 1. Scale: 0.2. 
A

$\begin{array}{lc} & \star \\ \text { ArthFT } & \text { LREYLHW } \\ \text { ArthTSF } & \text { QREYLHW } \\ \text { ArthTFL1 } & \text { LKEHLHW } \\ \text { ArthATC } & \text { LREHLHW } \\ \text { ArthBFT } & \text { MREYLHW } \\ \text { PtFT1 } & \text { LREYLHW } \\ \text { PtFT2 } & \text { LREYLHW } \\ \text { NEFT1 } & \text { LREYLHW } \\ \text { NEFT2 } & \text { LREYLHW } \\ \text { NEFT3 } & \text { QREYLHW } \\ \text { NtFT4 } & \text { LREYLHW } \\ \text { BVFT1 } & \text { QREYLHW } \\ \text { BVFT2 } & \text { LREYLHW }\end{array}$

B

\begin{tabular}{|c|c|}
\hline OnTWFT1B & KREH \\
\hline MaauFT1B_4 & CREHLHW \\
\hline CyenFT1B & REHLHW \\
\hline StpuFT1B & REHLHW \\
\hline GaelFT1B 1 & REHLHW \\
\hline DecaFT1B_1 & REHLHW \\
\hline PeFT-7 - & IREHLHW \\
\hline PheqFT1B_1 & CREHLHW \\
\hline PhluFT1B_1 & IREH LHW \\
\hline NezoFT1B & IREHLHW \\
\hline ApshFT1B & REHLHW \\
\hline MaauFT1B_2 & RELLLW \\
\hline MaauFT1B_3 & ERELLLW \\
\hline MacoFT1B & KRELLLW \\
\hline MayuFT1B & KRELLLW \\
\hline MaweFT1B & KRELLLW \\
\hline CatrFT1B & KRELLLW \\
\hline PeFT-4 & KRELLLW \\
\hline PheqFT1B_3 & KRE LLLW \\
\hline PhapFT1B & KRELLLW \\
\hline PhbeFT1B & KRELLLW \\
\hline PhluFT1B_2 & KRELLLW \\
\hline DecaFT1B_2 & IREHLLW \\
\hline OpspFT1B-1 & KREHLHW \\
\hline OpspFT1B_2 & KREHLHW \\
\hline CYfoFT1B & CREHLHW \\
\hline Vap1FT1B 3 & KRFHLHW \\
\hline AcFT3 & KREYLHW \\
\hline AcFT5 & REYLHW \\
\hline YubrFT1B & CREHLHW \\
\hline ZemaFT1B_1 & KREYLHW \\
\hline ZemaZCN19 & KREYLHW \\
\hline OrzaFT1B_1 & KREYLHW \\
\hline ZemazCN1 $\bar{\sigma}$ & KREYLHW \\
\hline SobiFT1B_1 & KREYLHW \\
\hline AFT1B 2 & REYLHV \\
\hline AncoFT1B 2 & KREYLHW \\
\hline ZemaZCN $2 \overline{0}$ & KREYLHV \\
\hline SobiFT1B_2 & CREYLHW \\
\hline ZemaFT1B 2 & KRENLHW \\
\hline ZemaZCN1 $1 \overline{7}$ & KRENLHV \\
\hline OrzaFT1B 3 & KREYLHV \\
\hline MaFT1 - & EREHLHY \\
\hline $\operatorname{Mat}$ & EREHLHV \\
\hline & EREYLHV \\
\hline & \\
\hline
\end{tabular}

C ArthFT
ArthTSF
ArthTFL1
ArthATC
ArthBFT
PtFT1
PtFT2
NtFT1
NtFT2
NtFT3
NtFT4
BVFT1
BVFT2

D

MaAuFT1A_3

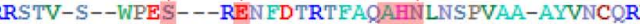
OnTWFT1A_1 .QRRGTV-S--VPGS---REN EDTRSEAQTHNLNSPVAI-AYVNCQRE CYENFT1A_1 1 QRRSTV-S--KPEL---REN EDTRTEAOAHNLNAPVAA-AYFNCORE PheqFT1A-1 QKRGTV-L--KPDS---REN EDTQTEAQANN LKTPVAATAYFNCQRE PeFT-1 - QKRGTV-L--KPDS---REN EDTQTEAQANN LKTPVAATAYFNCQRE PhapFT1A PKRGTV-L--KPDS---REN EDTQTEAQANN LKT PVAAAAYSTVS-PhluFT1A 2 .QKRGTV-P--KPDS---RENEDTRTEAQANN LKT PVAATAYFNCORE PhbeFT1A_1 'QKRGTV-L--KPDS---REN EDTRT EAQANN LKT PVAAAAYINCORE PhmoFT1A_1 QKRGTV-L--KPDS---REN EDTRTEAQANN LKT PVAAAAYINCORE MawEFTIA- QKKGTI-N-LKPNS---RENEDTQTEAQAYNLTTPAIT-AYFNCORF MaCOFT1A QKKGTI-N-LKPNS---RENEDTQTFAQAYNLTTPAIA-AYFNCQRE DeCaFT1A_1 'QRRSTV-S--KPDS---RENEDTRTEAQAYNLDSPVAA-AYFNCQRE DenoFT1A- . 'RRSTV-S--KPDS---RENEDTRTEAOAYNLDSPVAA-AYFNCQRF ElauFT1A EKRNSV-S--KPEL---REN ENTRTEAOVNNLS SPVAA-AYFNCQRE MirOFT1A_1 VRRNSV-S--SPET---RENESTQSEALEYDLSPPVAA-AYENCQRE OnTWFT1A 2 .VRRNSV-S--SPEK---RENFSTQSEALEYDLSPPVAA-AYFNCORE OnCIFT1A- LRRNSVLS--CPET---RENESTQSEALEYDLSPPVAA-AYENCORE CYenFT1A 2 HRRNSV-S--SPDT---REN FSTQSEALAYDLSSPVAA-AYFNCORE PheqFT1A_2 HRRNII-S--APNA---RENECTQAFADTYDLSEPVAA-AYFNCORE

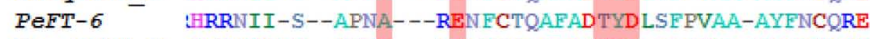
DeCaFT1A 2 .KRRTCV-Y--RPEV---RENFSTOSFAOEYELSSPVAA-AYFNCORE GOSCFT1A- 'EKRNNI-F-IEPEV---RENESTVTEAETENLS SPVAA-AYFNCQRE Vap1FT1A_1 MRRGTA-S--GPER---REN ENTRSEAELHNLGLPVGA-AYFNCORE Vap1FT1A 2 .LSRSMA-S--GPER---REN FNTRRFAELYNLGLPVGA-AYFNCORE MirOFT1A-2 LGNTV-F--EPEL---RHNECTLNEAOEYNLGMPVAA-VYFNCORF MaYuFT1A 2 LGRNTV-F--EPGL---RHNESTRSFATE-------LL-RLYSC--PhbeFT1A 2 -LGRNTV-F--EPEL---RHNFSTRDFAOEYNLTTSSLA--YFNCQRE PhmoFT1A_2 LGRNTV-F--EPEL---RHNESTRDFAQEYNLTTSSLA--YFNCQRE PhIUFT1A 3 -LGRNTV-F--EPEL---RHNESTRNEAOEYNLTTTSLA--YENCQRE PeFT-2 - LGRNTV-F--EPAL---RHNESTRNEAOEYNLTTESLA--YENCORE DeCaFT1A_3 LGRNTV-F--EPDL---RHNESTRSEAOEYNLSLPVAA-VYFNCORE VaplFT1A- 6 . LGRATV-F--APDV---RON FNIRAFAOQYN LGNAVAA-TYFNCORO NezOFT1A- MGRETV-F--APVM---RQNENTRSEAQEYNLGQPVAV-TYENCORQ ZemaFT1A_1 LGRETV-Y--APSR---RHN ESTRAFARRYNLGAPVAA-MYFNCQRQ SObIFT1A-1 -LGRETV-Y--APSW---RHNESTRGEARRYNLGAPVAA-MYENCORO SObIFT1A 2 . LGRDTV-F-AAPSR---RHN ENTRAFARRYNLGAPVAA-MFENCQRQ SobiFT1A_3 .LGRGTV-F--APQV---RQN FNLRNEARRFNLGKPVAA-MYFNCQRQ OrzaFT1A 2 . LGRTV-F--APQV---RONFNLRSEARR FN LGKPVAA-MYFNCORP MaFT3 - MGRGT-E--APQM---RHNFSTRRFAQQYYL-APVAA-TYFNCQRE MaFT4 MGRETV-F--TPEL---RHNESTRRFAMEHYL-VPVAA-TYYNCORE MaFT12 MGRTV-F--APOM---RHNFSTRRFAEOYYL-APVAA-TYYNCORE ZemaFT1A_2 :LGRGTV-F--APDM---RHN FNCKS FAROYHLDV-VAA-TYENCORE ZemaZCN1 $1 \overline{2}$ LGRGTV-F--APDM--RHN FNCKSEARQYHLDV-VAA-TYFNCORE SObIFT1A 4 -LGRTV-F--APDM---RHN FNCKNEARQYHLDI-VAA-TYFNCORE OrzaFT1A_3 LGRGTV-F--APEM---RHN FHCRSEAQQYHLDI-VAA-TYENCORE ZemaZCN8 - LGRGTV-E--APEM---RHNFNCRSFARQYHLSI-ATA-THFNCQRE OrZaFT1A 4 -LGRRTV-F--APEK---RHNFNCRIFAROHHLNI-VAA-TYFNCORE ACFT4 - LCKESV-F--APDV---RHN EDTRKFAREHYLGPPVAA-LYENCORE

FIGURE 5 | Sequence comparison between MonFT1A, MonFT1B and the canonical PEBP sequences from Arabidopsis thaliana and selected FT eudicot sequences with repressive function on key amino acids distinguishing FT and TFL1-like homologues (following Hanzawa et al., 2005; Ahn et al., 2006; Ho and Weigel, 2014). (A) Alignment of the positions homologous to Tyr-85 (Y)/His-88 (H) (in red and with upper star) from FT and TFL1 of $A$. thaliana respectively, as well as other repressive PEBP proteins from Beta vulgaris (Bv), Nicotiana tabacum (Nt) and Populus trichocarpa (Pt). (B) Same positions in the MonFT1B proteins. (C) Alignment of segments B and C of selected PEBP homologs, including canonical PEBP as well as FT homologs with repressive function. Blue dots (following Ahn et al., 2006) and red triangles (following critical residues identified by Ho and Weigel, 2014) indicate key amino acids distinguishing FT and TFL1-like homologs. (D) Same positions of segments B and C in the MonFT1A proteins. Red boxes on the sequence names indicate proteins with flowering promoter function, while the blue boxes indicate proteins with repressive function. 
used to distinguish between putative FT or TFL1 proteins (Ahn et al., 2006). When monocot FT proteins are compared in these positions, all MonFT proteins except for those belonging to MonFT1A show predictive residues of FT activity (IYN in Figures 5C,D and Supplementary Figure S3). Same positions in MonFT1A are highly variable and cannot be used to predict any functional activity by comparison with the canonical FT or TFL1 (Figures 5C,D).

Finally, between segment B and C, specific mutations in each of four critical residues, Glu-109 (E), Trp-138 (W), Gln140 (Q), and Asn-152 (N), can turn FT into a TFL1-like floral repressor (Ho and Weigel, 2014). When monocot FT proteins are compared in these positions, all MonFT proteins conserve predictive residues of FT promoting function except MonFT1A (WQN in Figures 5C,D and Supplementary Figure S3). These positions are highly variable in MonFT1A proteins, however, they have aminoacids more similar to TFL1 than to FT (Figures 5C,D).

\section{Expression Analyses}

In order to observe the expression patterns of the FT/TFL1like genes previously identified, we chose three orchid species: Cattleya trianae, Elleanthus aurantiacus and Gomphichis scaposa. These species were selected based on their low copy number of both FT and TFL1 genes. In addition, they represent two different orchid lineages, the Orchidoideae (G. scaposa) and the Epidendroideae (C. trianae and E. aurantiacus). Plants growing in nurseries (C. trianae) or in the wild (E. aurantiacus and G. scaposa) were dissected into leaves (L), vegetative meristem (SAM), inflorescence meristem (IM), and FB. In addition, for C. trianae expression was also evaluated in the pseudobulb (PS) and the axillary buds (AB). Amplifications by TD-PCR (Korbie and Mattick, 2008) at 30 cycles and 42 cycles were performed to evaluate minimal and maximal expression level during the vegetative-to-reproductive transition (Figure 6 and Supplementary Figure S4). In addition, qRT-PCR was done to verify RT-PCR results in a quantitative manner for $C$. trianae (Figure 7). However, expression analyses results presented here describe the 42 cycle RT-PCR and the qRT-PCR when available.

In Cattleya trianae RT-PCR allowed us to identify expression of CatrFT1B restricted to leaves (L), expression of CatrFT1C in axillary buds $(\mathrm{AB})$ and leaves (L), expression of CatrFT2A in the IM and broad expression of CatrTFL1 in PS, AB, L, IM, and FB (Figure 6A). qRT-PCR expression validates the RT-PCR analyses and in addition records the expression of CatrFT1B and CatrFT1C in IM (Figure 7).

RT-PCR in Elleanthus aurantiacus identifies the expression of ElauFT1A in all tissues dissected, especially in SAM and IM. Furthermore, ElauFT1C_2 and ElauFT2A_1 expression is detected in L, SAM and the IM, while ElauFT1A_2 is broadly expressed in L, SAM, IM, and FB (Figure 6B).

Finally, RT-PCR in Gomphichis scaposa results in the detection of GoscFT2A_1 in L, SAM, IM and BF, expression of GoscTFL1 in the SAM, the IM and the FB, and very low expression of GoscFT2C in the SAM. No expression is detected for GoscFT1A, GosFT1B, or GoscFT2A_2 (Figure 6C).

\section{DISCUSSION}

Previous phylogenetic analyses have divided the PEBP gene family in plants into the MFT-like, FT-like and TFL1-like subfamilies (Chardon and Damerval, 2005; Carmona et al., 2007; Danilevskaya et al., 2008; Hedman et al., 2009; Karlgren et al., 2011; Liu et al., 2016). Our ML results recover the same three groups, where the MFT-like grade is sister to the BFT/TFL1-like and FT-like clades (Supplementary Figure S1 and Figures 14). Monophyly and evolution of the MFT-like genes have been extensively debated (Hedman et al., 2009; Liu et al., 2016). In that respect, our analyses recover most MFT homologs from magnoliids, monocots and eudicots group with the canonical A. thaliana MFT. However, some MFT genes from few early divergent angiosperms and eudicots form a different group in the absence of $A$. thaliana homologs. It is likely that these genes belong to yet another subfamily that has been comparatively less sampled (Drabešová et al., 2016). Within the FT/TFL1like clades we were able to identify a number of large scale as well as local duplications and the associated changes in protein sequences in the resulting paralogs. Some had been previously identified (Chardon and Damerval, 2005; Carmona et al., 2007; Danilevskaya et al., 2008; Hedman et al., 2009; Karlgren et al., 2011; Lee et al., 2013; Liu et al., 2016; Chaurasia et al., 2017; Leeggangers et al., 2018) while some are reported here for the first time.

\section{The FT-Like Gene Subfamily Has Undergone Reiterative Duplication Events Compared to the TFL1-Like Subfamily in Monocots}

From the three PEBP subfamilies, the FT-like genes are by far the most diversified in terms of copy number (Chardon and Damerval, 2005; Danilevskaya et al., 2008; Lee et al., 2013; Chaurasia et al., 2017). Our ML analyses suggest that FT-like homologs have undergone a duplication event prior to the diversification of monocots and eudicots, giving rise to copies FT1 and FT2 (Figures 1-3). While FT1 genes, the A. thaliana FT orthologs, are widespread across angiosperms (Figures 1, 2), FT2 genes are retained exclusively in monocots (Figure 3). Monocot FT1 and FT2 genes have also duplicated extensively. We were able to recover two duplications in FT1 genes, resulting in the MonFT1A, MonFT1B, and MonFT1C paralogs, as well as two additional duplications in the FT2 genes, resulting in the MonFT2A, MonFT2B, and MonFT2C copies. These duplications coincide with whole genome duplication events that occurred during the evolution of monocots (Murat et al., 2017).

\section{Within FT-Like Genes the MonFT2 Homologs Are Likely Maintaining Their Roles as Flowering Promoters in Orchidaceae}

Orchidaceae species sampled have FT representatives in all six gene clades, in agreement with the five FT-like homologs reported for Phalaenopsis (Zhou et al., 2018). Most of the 


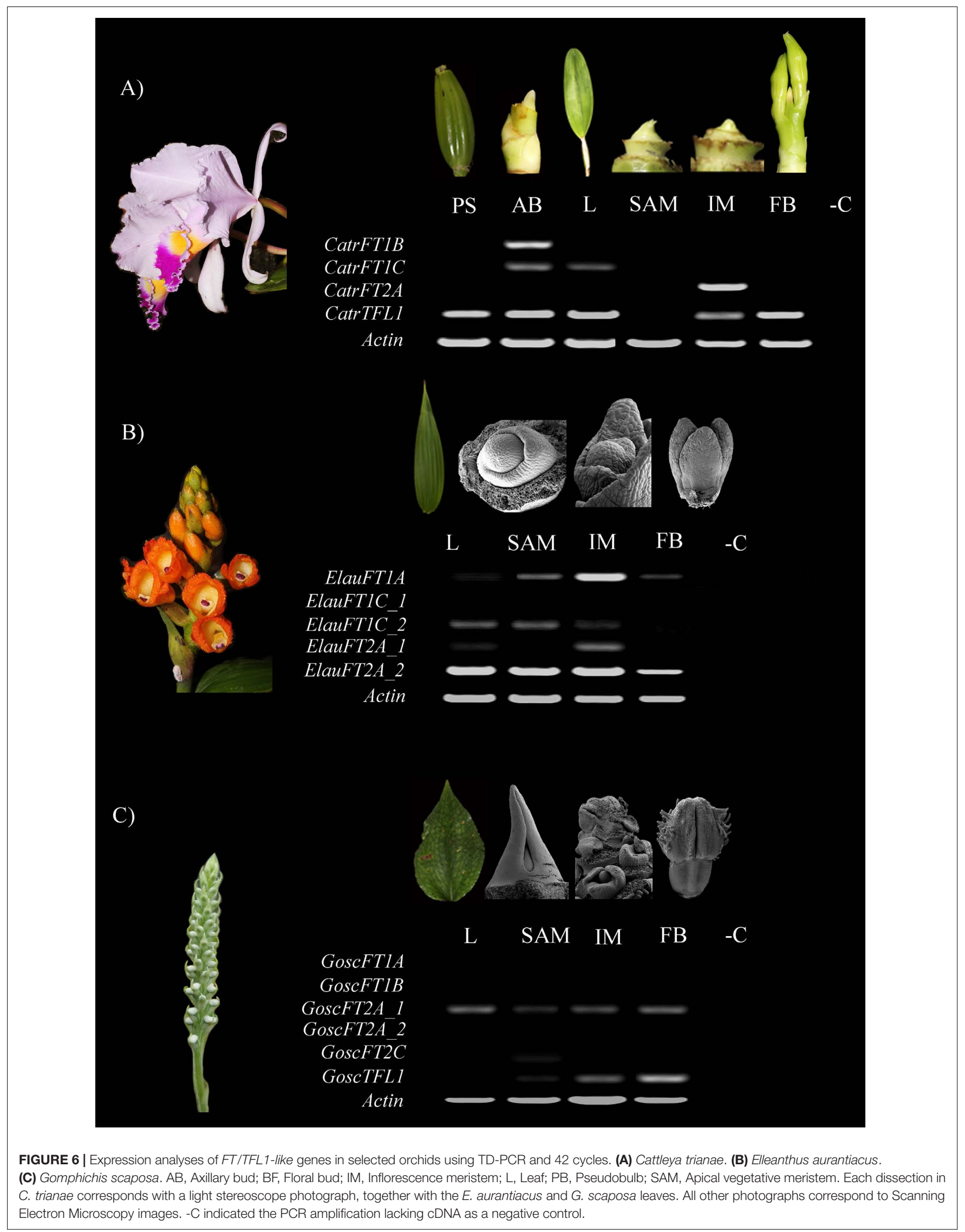




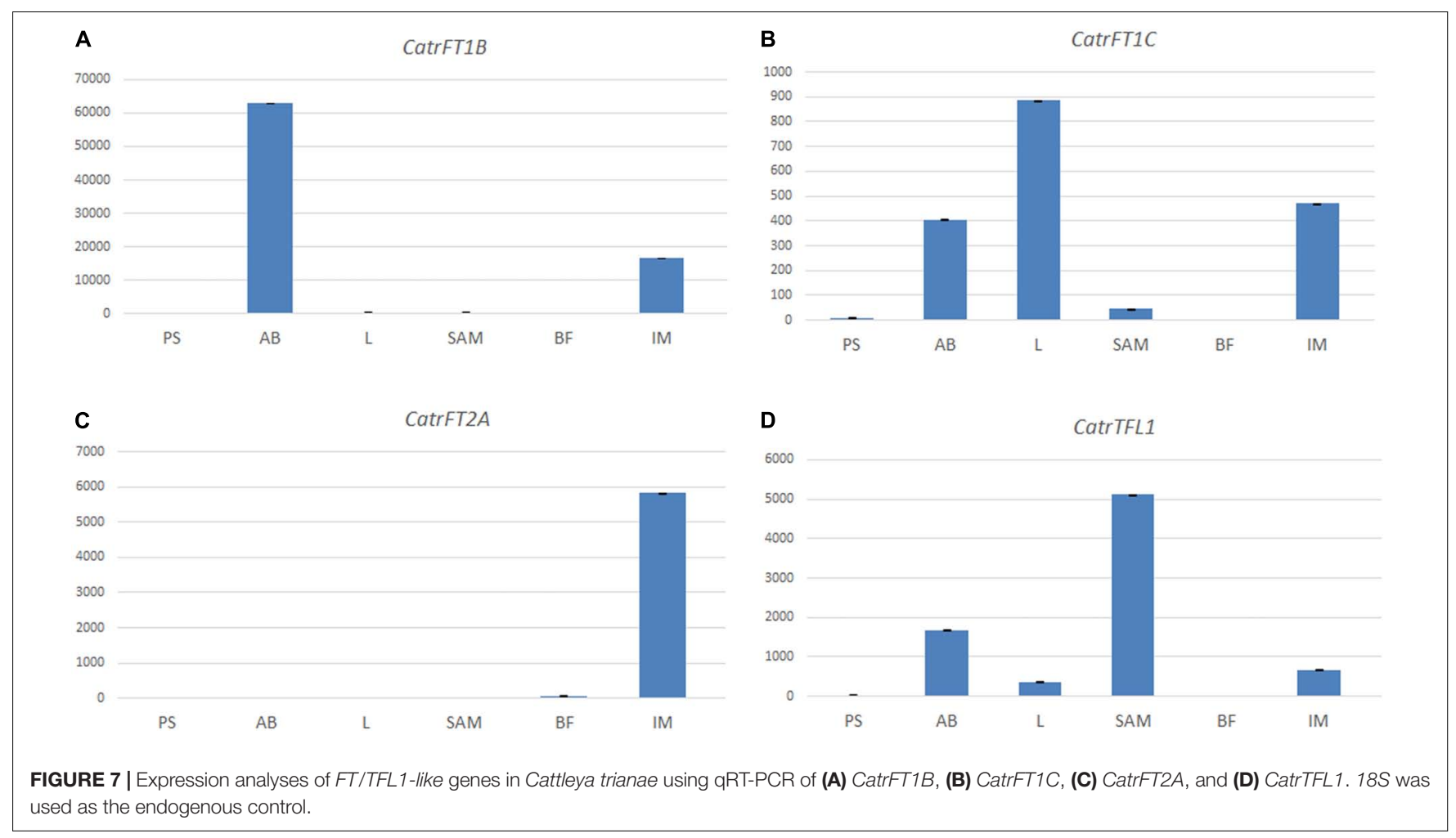

previously reported FT-like homologs in the family functionally analyzed (Hou and Yang, 2009; Huang W. et al., 2012; Li et al., 2012; Xiang et al., 2012; Li et al., 2014; Jang et al., 2015; Wang et al., 2017) nest in the MonFT2A clade in our analysis. Expression analyses of FT-like homologs from the FT2 clade have identified active transcription of MonFT2 members in leaves, SAM, IM, FBs, and fruits. These patterns are consistently found in Asparagales (Allium cepa), Liliales (Lilium longiflorum and Tulipa gesneriana), Orchidaceae (Cymbidium goeringii, Dendrobium "Chao Praya Smile," Dendrobium nobile, Oncidium Gower Ramsey, Phalaenopsis aphrodite, Phalaenopsis "Thailin Red Angel V31," Phalaenopsis "Fortune Saltzman”), Poales (Zea mays) and Zingiberales (Musa acuminata) (Danilevskaya et al., 2008; Hou and Yang, 2009; Li et al., 2012, 2014; Xiang et al., 2012; Lee et al., 2013; Jang et al., 2015; Manoharan et al., 2016; Chaurasia et al., 2017; Wang et al., 2017; Leeggangers et al., 2018; Zhou et al., 2018). In addition, FT2 homologs from $A$. cepa and Oncidium "Gower Ramsey" have also been detected in bulbs and pseudobulbs, respectively (Hou and Yang, 2009; Lee et al., 2013; Manoharan et al., 2016).

Functional analyses of MonFT2 representatives have uncovered their role in promoting flowering either endogenously (Kojima et al., 2002; Tamaki et al., 2007; Wang et al., 2017; Leeggangers et al., 2018), or by heterologous transformation in Arabidopsis thaliana, Nicotiana tabacum, or Oryza sativa (Hou and Yang, 2009; Huang W. et al., 2012; Xiang et al., 2012; Li et al., 2014; Jang et al., 2015; Leeggangers et al., 2018; Zhou et al., 2018). In addition, MonFT2 genes have been shown to induce bulb formation in Allium cepa (Lee et al., 2013). A single case has linked MonFT2 homologs with the negative regulation of flowering after the Tulipa gesneriana TgFT1 heterologous expression in A. thaliana (Leeggangers et al., 2018). In general, most MonFT2 transcripts are likely the functional homologs of the A. thaliana FT triggering vegetative-to-reproductive transition.

Our expression analyses shows that orchid MonFT2 orthologs are highly expressed compared to all other isolated $F T$ homologs. Interestingly, in the two terrestrial orchid species Elleanthus aurantiacus and Gomphichis scaposa at least one of the two MonFT2 homologs is broadly expressed in leaves, IM and FBs, while in the epiphytic Cattleya trianae, CatrFT2A is exclusively turned on in the IM. A detailed comparison of the orchid MonFT2 protein sequences (Supplementary Figure S3) with the canonical promoters and repressors in model core eudicots, shows that they are more likely to function as flowering promoters, like the homologs functionally evaluated in the same clade. Altogether, our data suggests that despite subtle differences in early expression in leaves, SAM and FBs among species with different habits, the role of MonFT2 homologs as positive flowering regulators seems to be retained in all orchids studied so far.

\section{Most MonFT1 Homologs in Orchidaceae Are Likely Repressing Flowering Transition With the Exception of MonFT1C Orthologs}

The expression of MonFT1 genes (i.e., those that nest in the MonFT1 clade in our analysis) has been less studied in orchids when compared to MonFT2 genes. MonFT1 transcripts have been 
isolated from leaves, IM as well as young and old FBs in Allium cepa (Asparagaceace), Tulipa gesneriana (Liliaceae), Phalaenopsis Tailin Red Angel V31 (Orchidaceae), Zea mays (Poaceae), and Musa acuminata (Musaceae) (Danilevskaya et al., 2008; Lee et al., 2013; Manoharan et al., 2016; Chaurasia et al., 2017; Leeggangers et al., 2018; Zhou et al., 2018). Only rarely they have been isolated from SAMs, stems and roots in T. gesneriana and Z. mays (Danilevskaya et al., 2008; Leeggangers et al., 2018), as well as from the bulb of A. cepa (Lee et al., 2013; Manoharan et al., 2016).

Functional data for MonFT1 are scarce when compared to data available for MonFT2 homologs. When overexpressed endogenously in Z. mays (Meng et al., 2011) or in a heterologous manner in A. thaliana, MonFT1 genes promote flowering (Lazakis et al., 2011; Chaurasia et al., 2017). However, additional experiments overexpressing the MonFT1 T. gesneriana homolog in $A$. thaliana have resulted in a slight delay in flowering (Leeggangers et al., 2018). Conversely, the overexpression of the $M$. acuminata MonFT1 homologs in A. thaliana has no effect on flowering time (Chaurasia et al., 2017). Finally, MonFT1 homologs can also regulate bulb formation in $A$. cepa (Lee et al., 2013; Rashid et al., 2019).

Our expression analyses show that MonFT1 orchid orthologs have a more restricted expression when compared to MonFT2 homologs, and sometimes, like in the case of Gomphichis scaposa expression is lacking from all the dissected organs. MonFT1 homologs from Cattleya trianae and Elleanthus aurantiacus can be detected in axillary buds, SAMs and IMs. Only MonFT1C homologs can be detected in leaves. Comparison of protein sequences among MonFT1 proteins with the canonical promoters and repressors in core eudicots points to MonFT1A and MonFT1B as negative regulators of the flowering transition, while MonFT1C more likely retain the role of promoting flowering. Thus, MonFT1A and MonFT1B might have been co-opted for the typical TFL1-like roles by delaying flowering and promoting inflorescence indeterminate meristem identity at least in Epidendroideae. This is particularly relevant as TFL1 homologs are not as abundant as FT genes in monocots (see below). From the MonFT1 genes, only MonFT1C genes are more likely functioning as flowering promoters in orchids as they accumulate in leaves and SAMs and IM, similar to what happens with the canonical FT from A. thaliana (Corbesier et al., 2007).

\section{TFL1-Like Genes Are Completely Lacking or Found in Low Number in Monocots When Compared to Eudicots}

Opposite to FT genes, the TFL1 subfamily has diversified more in eudicots than in monocots. Our analyses show an early duplication in angiosperms resulting in the TFL1-like and the BFT-like clades, in agreement with previous studies (Carmona et al., 2007; Liu et al., 2016). The BFT-like genes are present in most angiosperms and they are here proposed as an additional subfamily within the PEBP gene family. However, monocot representatives within this group are scarce suggesting they are likely undergoing pseudogenization. Our results also recover a eudicot specific duplication resulting in the EudiCEN-like and EudiTFL1-like copies (Figure 4). All monocot TFL1-like homologs are clustered in the MonTFL1-like clade (Figure 4) consistent to what has been previously reported (Liu et al., 2016; Gao et al., 2017).

Isolation of TFL1-like homologs from orchids has proven difficult and so far, only one homolog from Oncidium "Gower Ramsey" has been reported (Hou and Yang, 2009). Despite our sampling effort, TFL1-like homologs were found only in 12 orchid species, frequently as single copy with the exception of Vanilla planifolia, a species with two copies (Figure 4). No TFL1-like homologs were found in the genome of Phalaenopsis equestris (Cai et al., 2015). The lack of TFL1-like homologs in several orchid species may be either the result of the lack of expression of these genes on the sampled tissues, or a gradual loss-of-function of TFL1-like genes together with functional compensation by FTlike copies, specifically from the MonFT1A and MonFT1B clades (see above). In the latter scenario, it is possible that monocot specific FT-like copies have co-opted novel functions including flowering repression. However, functional analyses are necessary to test this hypothesis.

\section{The Remaining MonTFL1 Genes Likely Repress Flowering in Orchidaceae}

Some expression analyses have been performed for MonTFL1like homologs (i.e., those that nest in the MonTFL1 clade in our analysis) in Poaceae (Lolium perenne, Oryza sativa, Saccharum spp., and Zea mays). These genes are expressed in leaves, stems, roots, SAMs, IMs, and FBs (Jensen et al., 2001; Zhang et al., 2005; Danilevskaya et al., 2008; Coelho et al., 2014). MonTFL1-like genes in orchids have only been characterized in Oncidium "Gower Ramsey," where TFL1 is expressed in pseudobulbs and axillary buds (Hou and Yang, 2009). The overexpression of MonTFL1-like homologs in Z. mays delays flowering time and increases lateral branching and spikelet density in the inflorescence (Danilevskaya et al., 2010). Also, Oncidium MonTFL1-like can rescue the late flowering and the terminal flower in $t f l 1$ mutants from A. thaliana. Altogether, available data indicate that TFL1 monocot genes function similarly to their homologs in A. thaliana.

Our expression analyses show broad expression of TFL1-like homologs in all dissected organs from the epiphytic Cattleya trianae. On the other hand, in the terrestrial, understory specialist Gomphichis scaposa, TFL1-like genes are mainly found in the IM and the FBs. Finally, Elleanthus aurantiacus, a terrestrial species growing in full light lacks a MonTFL1 homolog. Thus, it is possible that copy number of MonTFL1 genes as well as their expression patterns vary in response to specific habitats. Altogether, evolution and expression patterns point to MonTFL1 orchid homologs as involved in flowering repression similar to the reports on TFL1 homolog from Oncidium "Gower Ramsey" (Hou and Yang, 2009).

\section{CONCLUSION}

FT-like genes are by far more diversified than TFL1-like genes in monocots, reaching up to six clades in the former when compared to a single lineage in the latter. Within MonFT1 genes, 
the comparative protein sequences of MonFT1A and MonFT1B suggest that they could have acquired functional roles in delaying flowering, a role assigned so far to TFL1-like proteins. This is further supported by the lack of expression from IMs seemingly uncoupled to the vegetative-to-reproductive transition. On the other hand, MonFT2 genes have retained their canonical motifs and roles in promoting flowering transition, with increasing expression levels from SAMs and leaves to IMs and FBs. Finally, TFL1-like genes are retained as single copy in orchids and can be even lacking from representative genomes (Phalaenopsis equestris) and transcriptomes sampled alike. Thus, they are likely undergoing selection toward pseudogenization. Their function could be linked to the parallel recruitment of MonFT1A and MonFT1B homologs in delaying flowering and maintaining indeterminacy in the inflorescence meristem. Our work also highlights the importance of large-scale genome and transcriptome analyses to build up a comprehensive framework for all FT/TFL1 monocot homologs simultaneously without focusing on specific gene clades. All functional hypotheses postulated here wait for functional validation in emerging model orchid species and comparative analyses in orchids with horticultural value.

\section{DATA AVAILABILITY STATEMENT}

The datasets generated for this study can be found in the NCBI GenBank accession MN968819-MN968822, MN968823-MN968828, MN968829-MN968836, MN968837MN968849, MN968850-MN968854, MN968855-MN968863, MN968864-MN968875, MN968876-MN968886, MN968887MN968888, MN968889-MN968890, MN968891-MN968897, MN968898-MN968901, MN968902-MN968907, MN968908MN968909, MN968910-MN968911, MN968912-MN968922, and MN968923-MN968926.

\section{REFERENCES}

Abascal, F., Zardoya, R., and Telford, M. J. (2010). TranslatorX: multiple alignment of nucleotide sequences guided by amino acid translations. Nucleic Acids Res. 38, 7-13. doi: 10.1093/nar/gkq291

Abe, M., Kobayashi, Y., Yamamoto, S., Daimon, Y., Yamaguchi, A., Ikeda, Y., et al. (2005). FD, a bZIP protein mediating signals from the floral pathway integrator FT at the shoot apex. Science 309, 1052-1056. doi: 10.1126/science.1115983

Ahn, J. H., Miller, D., Winter, V. J., Banfield, M. J., Lee, J. H., Yoo, S. Y., et al. (2006). A divergent external loop confers antagonistic activity on floral regulators FT and TFL1. EMBO J. 25, 605-614. doi: 10.1038/sj.emboj.7600950

Altschul, S. F., Gish, W., Miller, W., Myers, E. W., and Lipman, D. J. (1990). Basic local alignment search tool. J. Mol. Biol. 215, 403-410. doi: 10.1016/S00222836(05)80360-2

Alvarez, J., Guli, C. L., Yu, X. H., and Smyth, D. R. (1992). Terminal flower: a gene affecting inflorescence development in Arabidopsis thaliana. Plant J. 2, 103-116. doi: 10.1111/j.1365-313X.1992.00103.x

Amasino, R. (2010). Seasonal and developmental timing of flowering. Plant J. 61, 1001-1013. doi: 10.1111/j.1365-313X.2010.04148.x

Andrés, F., and Coupland, G. (2012). The genetic basis of flowering responses to seasonal cues. Nat. Rev. Genet. 13, 627-639. doi: 10.1038/nrg3291

Arango-Ocampo, C., González, F., Alzate, J. F., and Pabón-Mora, N. (2016). The developmental and genetic bases of apetaly in Bocconia frutescens (Chelidonieae: Papaveraceae). Evodevo 7:16. doi: 10.1186/s13227-016-0054-6

\section{AUTHOR CONTRIBUTIONS}

DO-Z, YM, and NP-M planned and designed the research, conducted fieldwork and performed the experiments. JA assembled the reference transcriptomes. All authors analyzed the data, wrote and approved the final manuscript.

\section{FUNDING}

This research was funded by CODI regionalización 2017 (code 2017-17146) and Estrategia de Sostenibilidad 2018-2019 from Universidad de Antioquia, the Convocatoria COLCIENCIAS 808-2018 (código 110180863819 CT 192-2019) and the 2019 BSA Graduate Student Research Award from the Botanical Society of America.

\section{ACKNOWLEDGMENTS}

We thank Victor Acosta, Francisco Villegas, and Olga Cardona for maintaining the floral material in greenhouse conditions. We thank Favio González (Universidad Nacional de Colombia) for careful revision of the manuscript and the Evo Devo UdeA group for laboratory assistance. We thank Markus Günther from the Technische Universität Dresden for technical assistance during SEM experiments.

\section{SUPPLEMENTARY MATERIAL}

The Supplementary Material for this article can be found online at: https://www.frontiersin.org/articles/10.3389/fpls.2020.00469/ full\#supplementary-material

Bailey, T. L., Boden, M., Buske, F. A., Frith, M., Grant, C. E., Clementi, L., et al. (2009). MEME SUITE: tools for motif discovery and searching. Nucleic Acids Res. 37, 202-208. doi: 10.1093/nar/gkp335

Benlloch, R., Berbel, A., Serrano-Mislata, A., and Madueño, F. (2007). Floral initiation and inflorescence architecture: a comparative view. Ann. Bot. 100, 659-676. doi: 10.1093/aob/mcm146

Blackman, B. K., Strasburg, J. L., Raduski, A. R., Michaels, S. D., and Rieseberg, L. H. (2010). The role of recently derived FT paralogs in Sunflower domestication. Curr. Biol. 20, 629-635. doi: 10.1016/j.cub.2010.01.059

Blázquez, M. A., Ahn, J. H., and Weigel, D. (2003). A thermosensory pathway controlling flowering time in Arabidopsis thaliana. Nat. Genet. 33, 168-171. doi: $10.1038 / \mathrm{ng} 1085$

Boss, P. K., Bastow, R. M., Mylne, J. S., and Dean, C. (2004). Multiple pathways in the decision to flower: enabling, promoting, and resetting. Plant Cell 16, 18-31. doi: 10.1105/tpc.015958

Bradley, D., Ratcliffe, O., Vincent, C., Carpenter, R., and Coen, E. (1997). Inflorescence commitment and architecture in Arabidopsis. Science 275, 80-83. doi: 10.1126/science.275.5296.80

Cai, J., Liu, X., Vanneste, K., Proost, S., Tsai, W. C., Liu, K. W., et al. (2015). The genome sequence of the orchid Phalaenopsis equestris. Nat. Genet. 47, 65-72. doi: $10.1038 / \mathrm{ng} .3149$

Carmona, M. J., Calonje, M., and Martínez-Zapater, J. M. (2007). The FT/TFL1 gene family in grapevine. Plant Mol. Biol. 63, 637-650. doi: 10.1007/s11103006-9113-z 
Chao, Y. T., Yen, S. H., Yeh, J. H., Chen, W. C., and Shih, M. C. (2017). Orchidstra 2.0-A Transcriptomics resource for the orchid family. Plant Cell Physiol. 58:e9. doi: $10.1093 / \mathrm{pcp} / \mathrm{pcw} 220$

Chardon, F., and Damerval, C. (2005). Phylogenomic analysis of the PEBP gene family in cereals. J. Mol. Evol. 61, 579-590. doi: 10.1007/s00239-004-0179-4

Chaurasia, A. K., Patil, H. B., Krischna, B., Subramanian, V. R., Sane, P. V., and Sane, A. P. (2017). Flowering time in banana (Musa spp.), a day neutral plant, is controlled by at least three FLOWERING LOCUS T homologues. Sci. Rep. 7:5935. doi: 10.1038/s41598-017-06118-x

Coelho, C. P., Minow, M. A., Chalfun-Júnior, A., and Colasanti, J. (2014). Putative sugarcane FT/TFL1 genes delay flowering time and alter reproductive architecture in Arabidopsis. Front. Plant Sci. 5:221. doi: 10.3389/fpls.2014. 00221

Conti, L., and Bradley, D. (2007). TERMINAL FLOWER 1 is a mobile signal controlling Arabidopsis architecture. Plant Cell 19, 767-778. doi: 10.1105/tpc. 106.049767

Corbesier, L., Vincent, C., Jang, S., Fornara, F., Fan, Q., Searle, I., et al. (2007). FT protein movement contributes to long-distance signaling in floral induction of Arabidopsis. Science 316, 1030-1033. doi: 10.1126/science.1141752

Danilevskaya, O. N., Meng, X., and Ananiev, E. V. (2010). Concerted modification of flowering time and inflorescence architecture by ectopic expression of TFL1like genes in maize. Plant Physiol. 153, 238-251. doi: 10.1104/pp.110.154211

Danilevskaya, O. N., Meng, X., Hou, Z., Ananiev, E. V., and Simmons, C. R. (2008). A genomic and expression compendium of the expanded PEBP gene family from maize. Plant Physiol. 146, 250-264. doi: 10.1104/pp.107.109538

Drabešová, J., Ėerná, L., Mašterová, H., Koloušková, P., Potock $\iota$, M., and Štorchová, H. (2016). The evolution of the FT/TFL1 genes in Amaranthaceae and their expression patterns in the course of vegetative growth and flowering in Chenopodium rubrum. G3 6, 3065-3076. doi: 10.1534/g3.116.028639

Fu, C. H., Chen, Y. W., Hsiao, Y. Y., Pan, Z. J., Liu, Z. J., Huang, Y. M., et al. (2011). OrchidBase: a collection of sequences of the transcriptome derived from orchids. Plant Cell Physiol. 52, 238-243. doi: 10.1093/pcp/pcq201

Gao, J., Huang, B., Wan, Y., Chang, J., Li, J., and Liao, P. (2017). Functional divergence and intron variability during evolution of angiosperm TERMINAL FLOWER1 (TFL1) genes. Sci. Rep. 7:14830. doi: 10.1038/s41598-017-13645-0

Hanano, S., and Goto, K. (2011). Arabidopsis TERMINAL FLOWER1 is involved in the regulation of flowering time and inflorescence development through transcriptional repression. Plant Cell 23, 3172-3184. doi: 10.1105/tpc.111. 088641

Hanzawa, Y., Money, T., and Bradley, D. (2005). A single amino acid converts a repressor to an activator of flowering. Proc. Natl. Acad. Sci. U.S.A. 102, 7748-7753. doi: 10.1073/pnas.0500932102

Harig, L., Beinecke, F. A., Oltmanns, J., Muth, J., Müller, O., Rüping, B., et al. (2012). Proteins from the FLOWERING LOCUS T-like subclade of the PEBP family act antagonistically to regulate floral initiation in tobacco. Plant J. 72, 908-921. doi: 10.1111/j.1365-313X.2012.05125.x

Hayama, R., Agashe, B., Luley, E., King, R., and Coupland, G. (2007). A circadian rhythm set by dusk determines the expression of FT homologs and the ShortDay photoperiodic flowering response in Pharbitis. Plant Cell 19, 2988-3000. doi: $10.1105 /$ tpc. 107.052480

Hedman, H., Källman, T., and Lagercrantz, U. (2009). Early evolution of the MFTlike gene family in plants. Plant Mol. Biol. 70, 359-369. doi: 10.1007/s11103009-9478-x

Henderson, I. R., and Dean, C. (2004). Control of Arabidopsis flowering: the chill before the bloom. Development 131, 3829-3838. doi: 10.1242/dev.01294

Ho, W. W., and Weigel, D. (2014). Structural features determining flowerpromoting activity of Arabidopsis FLOWERING LOCUS T. Plant Cell 26, 552-564. doi: 10.1105/tpc.113.115220

Hoang, D. T., Chernomor, O., von Haeseler, A., Minh, B. Q., and Vinh, L. S. (2018). UFBoot2: Improving the ultrafast bootstrap approximation. Mol. Biol. Evol. 35, 518-522. doi: 10.1093/molbev/msx281

Hou, C. J., and Yang, C. H. (2009). Functional analysis of FT and TFL1 orthologs from orchid (Oncidium Gower Ramsey) that regulate the vegetative to reproductive transition. Plant Cell Physiol. 50, 1544-1557. doi: 10.1093/pcp/ pcp099

Hsu, C. Y., Adams, J. P., Kim, H., No, K., Ma, C., Strauss, S. H., et al. (2011). FLOWERING LOCUS $T$ duplication coordinates reproductive and vegetative growth in perennial poplar. Proc. Natl. Acad. Sci. U.S.A. 108, 10756-10761. doi: 10.1073/pnas.1104713108

Hsu, H. F., Hsu, W. H., Lee, Y. I., Mao, W. T., Yang, J. Y., Li, J. Y., et al. (2015). Model for perianth formation in orchids. Nat. Plants 1:15046. doi: 10.1038/ nplants. 2015.46

Huang, W., Fang, Z., Zeng, S., Zhang, J., Wu, K., Chen, Z., et al. (2012). Molecular cloning and functional analysis of three FLOWERING LOCUS T (FT) homologous genes from Chinese Cymbidium. Int. J. Mol. Sci. 13, 1138511398. doi: 10.3390/ijms130911385

Huang, N. C., Jane, W. N., Chen, J., and Yu, T. S. (2012). Arabidopsis thaliana CENTRORADIALIS homologue (ATC) acts systemically to inhibit floral initiation in Arabidopsis. Plant J. 72, 175-184. doi: 10.1111/j.1365-313X.2012. 05076.x

Imamura, T., Nakatsuka, T., Higuchi, A., Nishihara, M., and Takahashi, H. (2011). The gentian orthologs of the FT/TFL1 gene family control floral initiation in Gentiana. Plant Cell Physiol. 52, 1031-1041. doi: 10.1093/pcp/pcr055

Jang, S., Choi, S. C., Li, H. Y., An, G., and Schmelzer, E. (2015). Functional characterization of Phalaenopsis aphrodite flowering genes PaFT1 and PaFD. PLoS One 10:e0134987. doi: 10.1371/journal.pone.0134987

Jang, S., Torti, S., and Coupland, G. (2009). Genetic and spatial interactions between FT, TSF and SVP during the early stages of floral induction in Arabidopsis. Plant J. 60, 614-625. doi: 10.1111/j.1365-313X.2009.03986.x

Jensen, C. S., Salchert, K., and Nielsen, K. K. (2001). A TERMINAL FLOWER1-Like gene from perennial ryegrass involved in floral transition and axillary meristem identity. Plant Physiol. 125, 1517-1528. doi: 10.1104/pp.125.3.1517

Kalyaanamoorthy, S., Minh, B. Q., Wong, T. K. F., von Haeseler, A., and Jermiin, L. S. (2017). ModelFinder: fast model selection for accurate phylogenetic estimates. Nat. Methods 14, 587-589. doi: 10.1038/nmeth.4285

Kardailsky, I., Shukla, V. K., Ahn, J. H., Dagenais, N., Christensen, S. K., Nguyen, J. T., et al. (1999). Activation tagging of the floral inducer FT. Science 286, 1962-1965. doi: 10.1126/science.286.5446.1962

Karlgren, A., Gyllenstrand, N., Källman, T., Sundström, J. F., Moore, D., Lascoux, M., et al. (2011). Evolution of the PEBP gene family in plants: functional diversification in seed plant evolution. Plant Physiol. 156, 1967-1977. doi: 10. 1104/pp.111.176206

Kobayashi, Y., Kaya, H., Goto, K., Iwabuchi, M., and Araki, T. (1999). A pair of related genes with antagonistic roles in mediating flowering signals. Science 286, 1960-1962. doi: 10.1126/science.286.5446.1960

Kojima, S., Takahashi, Y., Kobayashi, Y., Monna, L., Sasaki, T., Araki, T., et al. (2002). Hd3a, a rice ortholog of the Arabidopsis FT gene, promotes transition to flowering downstream of Hdl under Short-Day conditions. Plant Cell Physiol. 43, 1096-1105. doi: 10.1093/pcp/pcf156

Koornneef, M., Hanhart, C. J., and van der Veen, J. H. (1991). A genetic and physiological analysis of late flowering mutants in Arabidopsis thaliana. Mol. Gen. Genet. 229, 57-66. doi: 10.1007/BF00264213

Korbie, D., and Mattick, J. (2008). Touchdown PCR for increased specificity and sensitivity in PCR amplification. Nat. Protoc. 3, 1452-1456. doi: 10.1038/nprot. 2008.133

Lazakis, C. M., Coneva, V., and Colasanti, J. (2011). ZCN8 encodes a potential orthologue of Arabidopsis FT florigen that integrates both endogenous and photoperiod flowering signals in maize. J. Exp. Bot. 62, 4833-4842. doi: 10.1093/ jxb/err129

Lee, J., and Lee, I. (2010). Regulation and function of SOC1, a flowering pathway integrator. J. Exp. Bot. 61, 2247-2254. doi: 10.1093/jxb/erq098

Lee, R., Baldwin, S., Kenel, F., McCallum, J., and Macknight, R. (2013). FLOWERING LOCUS T genes control onion bulb formation and flowering. Nat. Commun. 4:2884. doi: 10.1038/ncomms3884

Leeggangers, H. A. C. F., Rosilio-Brami, T., Bigas-Nadal, J., Rubin, N., van Dijk, A. D. J., Núñez de Cáceres, F., et al. (2018). Tulipa gesneriana and Lilium longiflorum PEBP genes and their putative roles in flowering time control. Plant Cell Physiol. 59, 90-106. doi: 10.1093/pcp/pcx164

Li, D. M., Lu, F. B., Zhu, G. F., Sun, Y. B., Liu, H. L., Liu, J. W., et al. (2014). Molecular characterization and functional analysis of a Flowering locus $T$ homolog gene from a Phalaenopsis orchid. Genet. Mol. Res. 13, 5982-5994. doi: $10.4238 / 2014$

Li, R., Wang, A., Sun, S., Liang, S., Wang, X., Ye, Q., et al. (2012). Functional characterization of FT and MFT ortholog genes in orchid (Dendrobium nobile 
Lindl) that regulate the vegetative to reproductive transition in Arabidopsis. Plant Cell Tissue Organ. Cult. 111, 143-151. doi: 10.1007/s11240-012-0178-x

Lifschitz, E., Ayre, B. G., and Eshed, Y. (2014). Florigen and anti-florigen - A systemic mechanism for coordinating growth and termination in flowering plants. Front. Plant Sci. 5:465. doi: 10.3389/fpls.2014.00465

Lin, M. K., Belanger, H., Lee, Y. J., Varkonyi-Gasic, E., Taoka, K., Miura, E., et al. (2007). FLOWERING LOCUS T protein may act as the long-distance florigenic signal in the cucurbits. Plant Cell 19, 1488-1506. doi: 10.1105/tpc.107.051920

Liu, C., Thong, Z., and Yu, H. (2009). Coming into bloom: the specification of floral meristems. Development 136, 3379-3391. doi: 10.1242/dev.033076

Liu, Y. Y., Yang, K. Z., Wei, X. X., and Wang, X. Q. (2016). Revisiting the phosphatidylethanolamine-binding protein (PEBP) gene family reveals cryptic FLOWERING LOCUS $T$ gene homologs in gymnosperms and sheds new light on functional evolution. New Phytol. 12, 730-744. doi: 10.1111/nph.14066

Livak, K. J., and Schmittgen, T. D. (2001). Analysis of relative gene expression data using real-time quantitative PCR and the 2- $\Delta \Delta$ CT method. Methods 52, 402-408. doi: 10.1006/meth.2001.1262

Lu, K. J., Huang, N. C., Liu, Y. S., Lu, C. A., and Yu, T. S. (2012). Longdistance movement of Arabidopsis FLOWERING LOCUS T RNA participates in systemic floral regulation. RNA Biol. 9, 653-662. doi: 10.4161/rna.19965

Madrigal, Y., Alzate, J. F., and Pabón-Mora, N. (2017). Evolution and expression patterns of TCP genes in Asparagales. Front. Plant Sci. 8:9. doi: 10.3389/fpls. 2017.00009

Manoharan, R. K., Han, J. S., Vijayakumar, H., Subramani, B., Thamilarasan, S. K., Park, J. I., et al. (2016). Molecular and functional characterization of FLOWERING LOCUS T homologs in Allium cepa. Molecules 21:E217. doi: $10.3390 /$ molecules 21020217

Meng, X., Muszynski, M. G., and Danilevskaya, O. N. (2011). The FT-Like ZCN8 gene functions as a floral activator and is involved in photoperiod sensitivity in maize. Plant Cell 23, 942-960. doi: 10.1105/tpc.110.081406

Mouradov, A., Cremer, F., and Coupland, G. (2002). Control of flowering time: interacting pathways as a basis for diversity. Plant Cell 14, 111-130. doi: 10. $1105 /$ tpc.001362

Murat, F., Armero, A., Pont, C., Klopp, C., and Salse, J. (2017). Reconstructing the genome of the most recent common ancestor of flowering plants. Nat. Genet. 49, 490-496. doi: 10.1038/ng.3813

Mutasa-Göttgens, E., and Hedden, P. (2009). Gibberellin as a factor in floral regulatory networks. J. Exp. Bot. 60, 1979-1989. doi: 10.1093/jxb/erp040

Nan, H., Cao, D., Zhang, D., Li, Y., Lu, S., Tang, L., et al. (2014). GmFT2a and GmFT5 $a$ redundantly and differentially regulate flowering through interaction with and upregulation of the bZIP transcription factor GmFDL19 in soybean. PLoS One 9:e97669. doi: 10.1371/journal.pone.0097669

Navarro, C., Abelenda, J. A., Cruz-Oró, E., Cuéllar, C. A., Tamaki, S., Silva, J., et al. (2011). Control of flowering and storage organ formation in potato by FLOWERING LOCUS T. Nature 478, 119-122. doi: 10.1038/nature 10431

Nguyen, L. T., Schmidt, H. A., von Haeseler, A., and Minh, B. Q. (2015). IQ-TREE: a fast and effective stochastic algorithm for estimating maximum-likelihood phylogenies. Mol. Biol. Evol. 32, 268-274. doi: 10.1093/molbev/msu300

Ortiz-Ramírez, C. I., Plata-Arboleda, S., and Pabón-Mora, N. (2018). Evolution of genes associated with gynoecium patterning and fruit development in Solanaceae. Ann. Bot. 121, 1211-1230. doi: 10.1093/aob/mcy007

Pabón-Mora, N., and González, F. (2008). Floral ontogeny of Telipogon spp. (Orchidaceae) and insights on the perianth symmetry in the family. Int. J. Plant Sci. 169, 1159-1173. doi: 10.1086/591982

Pabón-Mora, N., Suárez-Barón, H., Ambrose, B. A., and González, F. (2015). Flower development and perianth identity candidate genes in the basal angiosperm Aristolochia fimbriata (Piperales: Aristolochiaceae). Front. Plant Sci. 6:1095. doi: 10.3389/fpls.2015.01095

Pérez-Ruiz, R. V., García-Ponce, B., Marsch-Martínez, N., Ugartechea-Chirino, Y., Villajuana-Bonequi, M., de Folter, S., et al. (2015). XAANTAL2 (AGL14) is an important component of the complex gene regulatory network that underlies Arabidopsis shoot apical meristem transitions. Mol. Plant 8, 796-813. doi: 10. 1016/j.molp.2015.01.017

Pin, P. A., Benlloch, R., Bonnet, D., Wremerth-Weich, E., Kraft, T., Gielen, J. J., et al. (2010). An antagonistic pair of FT homologs mediates the control of flowering time in Sugar Beet. Science 330, 1397-1400. doi: 10.1126/science. 1197004
Randoux, M., Davière, J. M., Jeauffre, J., Thouroude, T., Pierre, S., Toualbia, Y., et al. (2014). RoKSN, a floral repressor, forms protein complexes with RoFD and RoFT to regulate vegetative and reproductive development in rose. New Phytol. 202, 161-173. doi: 10.1111/nph.12625

Rashid, M. H. A., Cheng, W., and Thomas, B. (2019). Temporal and spatial expression of Arabidopsis gene homologs control day length adaptation and bulb formation in Onion (Allium cepa L.). Sci. Rep. 9:14629. doi: 10.1038/ s41598-019-51262-1

Ratcliffe, O. J., Amaya, I., Vincent, C. A., Rothstein, S., Carpenter, R., Coen, E. S., et al. (1998). A common mechanism controls the life cycle and architecture of plants. Develoment 125, 1609-1615.

Ratcliffe, O. J., Bradley, D. J., and Coen, E. S. (1999). Separation of shoot and floral identity in Arabidopsis. Development 126, 1109-1120.

Shannon, S., and Meeks-Wagner, D. R. (1991). A mutation in the Arabidopsis TFL1 gene affects inflorescence meristem development. Plant Cell 3, 877-892. doi: 10.1105/tpc.3.9.877

Suárez-Baron, H., Alzate, J. F., González, F., Ambrose, B. A., and Pabón-Mora, N. (2019). Genetic mechanisms underlying perianth epidermal elaboration of Aristolochia ringens Vahl (Aristolochiaceae). Flora 253, 56-66. doi: 10.1016/j. flora.2019.03.004

Suárez-López, P., Wheatley, K., Robson, F., Onouchi, H., Valverde, F., and Coupland, G. (2001). CONSTANS mediates between the circadian clock and the control of flowering in Arabidopsis. Nature 410, 1116-1120. doi: 10.1038/ 35074138

Tamaki, S., Matsuo, S., Wong, H. L., Yokoi, S., and Shimamotof, K. (2007). Hd3a protein is a mobile flowering signal in rice. Science 316, 1033-1036. doi: 10. $1126 /$ science. 1141753

Taoka, K., Ohki, I., Tsuji, H., Furuita, K., Hayashi, K., Yanase, T., et al. (2011). 143-3 proteins act as intracellular receptors for rice Hd3a florigen. Nature 476, 332-335. doi: 10.1038/nature10272

Taoka, K., Ohki, I., Tsuji, H., Kojima, C., and Shimamoto, K. (2013). Structure and function of florigen and the receptor complex. Trends Plant Sci. 18, 287-294. doi: 10.1016/j.tplants.2013.02.002

Teixeira da Silva, J. A. (2013). Orchids: advances in tissue culture, genetics, phytochemistry and transgenic biotechnology. Floricult. Ornament. Biotechnol. 7, 1-52.

Teixeira da Silva, J. A., Aceto, S., Liu, W., Yu, H., and Kanno, A. (2014). Genetic control of flower development, color and senescence of Dendrobium orchids. Sci. Hortic. 175, 74-86. doi: 10.1016/j.scienta.2014.05.008

Teo, C. J., Takahashi, K., Shimizu, K., Shimamoto, K., and Taoka, K. I. (2017). Potato tuber induction is regulated by interactions between components of a tuberigen complex. Plant Cell Physiol. 58, 365-374. doi: 10.1093/pcp/pcw197

Thouet, J., Quinet, M., Lutts, S., Kinet, J. M., and Périlleux, C. (2012). Repression of floral meristem fate is crucial in shaping tomato inflorescence. PLoS One 7:e31096. doi: 10.1371/journal.pone.0031096

Valverde, F. (2011). CONSTANS and the evolutionary origin of photoperiodic timing of flowering. J. Exp. Bot. 62, 2453-2463. doi: 10.1093/jxb/erq449

Wang, Y., Liu, L., Song, S., Li, Y., Shen, L., and Yu, H. (2017). DOFT and DOFTIP1 affect reproductive development in the orchid Dendrobium Chao Praya Smile. J. Exp. Bot. 68, 5759-5772. doi: 10.1093/jxb/erx400

Wang, Z., Zhou, Z., Liu, Y., Liu, T., Li, Q., and Ji, Y. (2015). Functional evolution of phosphatidylethanolamine binding proteins in Soybean and Arabidopsis. Plant Cell 27, 323-336. doi: 10.1105/tpc.114.135103

Wellmer, F., and Riechmann, J. L. (2010). Gene networks controlling the initiation of flower development. Trends Genet. 26, 519-527. doi: 10.1016/j.tig.2010.09. 001

Wigge, P. A., Kim, M. C., Jaeger, K. E., Busch, W., Schmid, M., Lohmann, J. U., et al. (2005). Integration of spatial and temporal information during floral induction in Arabidopsis. Science 309, 1056-1059. doi: 10.1126/science.1114358

Xiang, L., Li, X., Qin, D., Guo, F., Wu, C., Miao, L., et al. (2012). Functional analysis of FLOWERING LOCUS T orthologs from spring orchid (Cymbidium goeringii Rchb. f.) that regulates the vegetative to reproductive transition. Plant Physiol. Biochem. 58, 98-105. doi: 10.1016/j.plaphy.2012.06.011

Yamaguchi, A., Kobayashi, Y., Goto, K., Abe, M., and Araki, T. (2005). TWIN SISTER OF FT (TSF) acts as a floral pathway integrator redundantly with FT. Plant Cell Physiol. 46, 1175-1189. doi: 10.1093/pcp/pci151

Yoo, S. J., Chung, K. S., Jung, S. H., Yoo, S. Y., Lee, J. S., and Ahn, J. H. (2010). BROTHER OF FT AND TFL1 (BFT) has TFL1-like activity and functions 
redundantly with TFL1 in inflorescence meristem development in Arabidopsis. Plant J. 63, 241-253. doi: 10.1111/j.1365-313X.2010.04234.x

Yoo, S. Y., Kardailsky, I., Lee, J. S., Weige, D., and Ahn, J. H. (2004). Acceleration of flowering by overexpression of MFT (MOTHER OF FT AND TFL1). Mol. Cells $17,95-101$.

Zhang, S., Hu, W., Wang, L., Lin, C., Cong, B., Sun, C., et al. (2005). TFL1/CEN-like genes control intercalary meristem activity and phase transition in rice. Plant Sci. 168, 1393-1408. doi: 10.1016/j.plantsci.2004.10.022

Zhou, S., Jiang, L., Guan, S., Gao, Y., Gao, Q., Wang, G., et al. (2018). Expression profiles of five FT-like genes and functional analysis of $P h F T-1$ in a Phalaenopsis hybrid. Electron. J. Biotechnol. 31, 75-83. doi: 10.1016/j.ejbt.2017.11.003
Conflict of Interest: The authors declare that the research was conducted in the absence of any commercial or financial relationships that could be construed as a potential conflict of interest.

Copyright (c) 2020 Ospina-Zapata, Madrigal, Alzate and Pabón-Mora. This is an open-access article distributed under the terms of the Creative Commons Attribution License (CC BY). The use, distribution or reproduction in other forums is permitted, provided the original author(s) and the copyright owner(s) are credited and that the original publication in this journal is cited, in accordance with accepted academic practice. No use, distribution or reproduction is permitted which does not comply with these terms. 